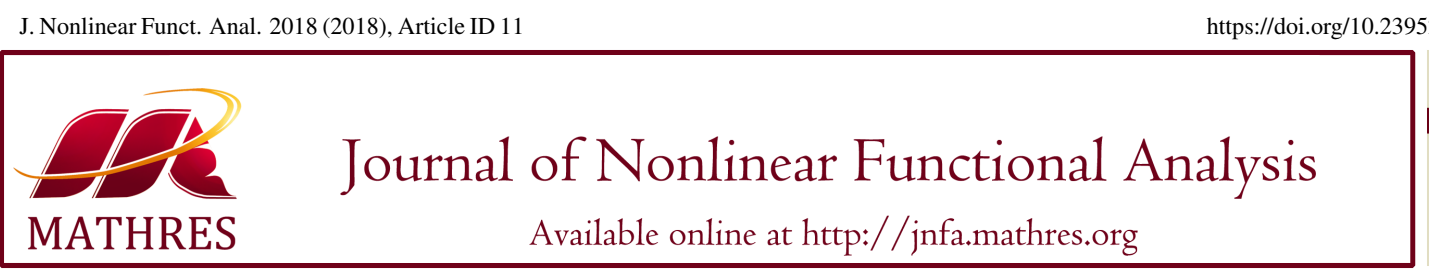

https://doi.org/10.23952/jnfa.2018.11

\title{
$p(x)$-LAPLACIAN-LIKE PROBLEMS WITH NEUMANN CONDITION ORIGINATED FROM A CAPILLARY PHENOMENA
}

\author{
SHAPOUR HEIDARKHANI*, AMJAD SALARI \\ Department of Mathematics, Faculty of Sciences, Razi University, 67149 Kermanshah, Iran
}

\begin{abstract}
In this paper, we study the multiplicity results for $p(x)$-Laplacian-like problems with Neumann condition, originated from a capillary phenomena. Using variational methods and the critical point theory, the existence of two and three solutions for the problem is discussed.
\end{abstract}

Keywords. Critical point theory; Multiple solutions; Neumann condition; Variable exponent Sobolev space; Variational method.

2010 Mathematics Subject Classification. 35J20, 35J60.

\section{INTRODUCTION}

Let $\Omega \subset \mathbb{R}^{N}$ be an open bounded domain with smooth boundary $\partial \Omega$. In this paper, we are mainly concerned with existence and multiplicity results for the $p(x)$-Laplacian-like problem, originated from a capillary phenomena,

$$
\left\{\begin{array}{lll}
-\operatorname{div}\left(\left(1+\frac{|\nabla u|^{p(x)}}{\sqrt{1+|\nabla u|^{2 p(x)}}}\right)|\nabla u|^{p(x)-2} \nabla u\right)+\alpha(x)|u|^{p(x)-2} u & \\
=\lambda f(x, u), & \text { in } \Omega, \\
\frac{\partial u}{\partial v}=0, & \text { on } \partial \Omega,
\end{array}\right.
$$

where $\Omega \subset \mathbb{R}^{N}(N \geq 2)$ is a bounded domain with boundary of class $C^{1}, v$ is the outer unit normal to $\partial \Omega, \lambda>0, \mu \geq 0, \alpha \in \mathrm{L}^{\infty}(\Omega)$ with $\operatorname{essinf}_{\Omega} \alpha \geq 0, f: \Omega \times \mathbb{R} \rightarrow \mathbb{R}$ is an $\mathrm{L}^{1}$-Carathéodory function and $p \in \mathrm{C}^{0}(\bar{\Omega})$ satisfies the condition

$$
N<p^{-}:=\inf _{x \in \bar{\Omega}} p(x) \leq p^{+}:=\sup _{x \in \bar{\Omega}} p(x)<+\infty .
$$

During the last fifteen years, differential and partial differential equations with variable exponent growth conditions have become increasingly popular. This is partly due to their frequent appearance in applications such as the modeling of electrorheological fluids, image restoration, elastic mechanics and

${ }^{*}$ Corresponding author.

E-mail addresses: s.heidarkhani@razi.ac.ir (S. Heidarkhani), amjads45@yahoo.com (A. Salari).

Received October 16, 2017; Accepted February 13, 2018.

(C)2018 Journal of Nonlinear Functional Analysis 
continuum mechanics, and these problems are also very interesting from a purely mathematical point of view as well. Recently, Fan and Deng [1] studied the existence and multiplicity of positive solutions for the inhomogeneous Neumann boundary value problem involving the $p(x)$-Laplacian of the following form

$$
\begin{cases}-\operatorname{div}\left(|\nabla u|^{p(x)-2} \nabla u\right)+\lambda|u|^{p(x)-2} u=f(x, u), & \text { in } \Omega, \\ |\nabla u|^{p(x)-2} \frac{\partial u}{\partial \eta}=\varphi, & \text { on } \partial \Omega,\end{cases}
$$

where $\Omega$ is a bounded smooth domain in $\mathbb{R}^{N}, p \in \mathrm{C}^{1}(\Omega)$ and $p(x)>1$ for $x \in \Omega, \varphi \in \mathrm{C}^{0, \gamma}(\partial \Omega)$ with $\gamma \in(0,1), \varphi \geq 0$ and $\varphi \neq 0$ on $\partial \Omega$. They, under appropriate assumptions on $f$, obtained that there exists $\lambda_{*}>0$ such that the problem has at least two positive solutions if $\lambda>\lambda_{*}$, has at least one positive solution if $\lambda=\lambda_{*}$, and has no positive solution if $\lambda<\lambda_{*}$. Deng [2], based on a local mountain pass theorem without (P.S) condition and Ricceri's variational principle, obtained the existence and multiplicity of non-trivial solutions for the following $p(x)$-Laplacian double perturbed Neumann problem with nonlinear boundary condition

$$
\begin{cases}-\Delta_{p(x)} u+a(x)|u|^{p(x)-2} u=f(x, u)+\lambda h_{1}(x, u), & \text { in } \Omega \\ |\nabla u|^{p(x)-2} \frac{\partial u}{\partial \gamma}=g(x, u)+\mu h_{2}(x, u), & \text { on } \partial \Omega\end{cases}
$$

where $\Omega$ is a bounded open domain in $\mathbb{R}^{N}$ with smooth boundary, $-\Delta_{p(x)} u=-\operatorname{div}\left(|\nabla u|^{p(x)-2} \nabla u\right)$ is the $p(x)$-Laplacian with $p \in \mathrm{C}(\bar{\Omega}), p(x)>1, \lambda, \mu \in \mathbb{R}, a \in \mathrm{L}^{\infty}(\Omega)$ with $\operatorname{essinf}_{x \in \Omega} a(x)=a^{-}>0$, and $\gamma$ is the outward unit normal to $\partial \Omega$. Cammaroto and Vilasi [3], based on a recent variational principle due to Ricceri, established the existence of at least three solutions for the Neumann problem involving the $p(x)$-Laplacian operator. In [4], the existence of at least three solutions for elliptic problems driven by a $p(x)$-Laplacian was established based on the variational methods and critical-point theory. The existence of at least one nontrivial solution was also proved.

Problem $\left(P_{\lambda}^{f}\right)$ is the developed form of the elliptic Dirichlet problem

$$
\begin{cases}-\operatorname{div}\left(\frac{\nabla u}{\sqrt{1+|\nabla u|^{2}}}\right)=f(t, u) & \text { in } \Omega, \\ u=0 & \text { on } \partial \Omega\end{cases}
$$

where $\Omega \subseteq \mathbb{R}^{N}(N \geq 2)$ is a bounded open subset with sufficiently smooth boundary $\partial \Omega$ and $f: \Omega \times \mathbb{R} \rightarrow$ $\mathbb{R}$ is a suitable Carathéodory function. It is well-known that a solution $u$ of (1.1) defines a Cartesian surface in $\mathbb{R}^{N+1}$ whose mean curvature is prescribed by the right hand side of the equation and this problem plays an important role in differential geometry and in the theory of relativity.

Existence, non-existence and multiplicity of positive solutions of problem (1.1) have been studied recently. We just observe that the one-dimensional problem has been rather thoroughly discussed, by using different methods; see $[5,6,7,8]$ and references therein. In particular, if $f(x, u)=\lambda u^{p}$, where $p>0$ is given and $\lambda>0$ is a parameter, the existence of positive solutions of (1.1) was established assuming $p>1$ (the convex, or superlinear, case), with $p<\frac{N+2}{N-2}$ if $N>2$, and $\lambda$ large [9], or $p=1$ (the linear case) and $\lambda$ in a left neighborhood of the principal eigenvalue $\lambda_{1}$ of $\Delta$ in $\mathrm{H}_{0}^{1}(\Omega)$ [10], or $p<1$ (the concave, or sublinear, case) and $\lambda$ small [11]. The solutions found in these papers belong at least to $H_{0}^{1}(\Omega)$ and are intended in the usual weak sense. In [12], the existence of a second positive solution has been proved in the concave case when $\lambda$ is small, assuming further $p \in] 0, \frac{1}{N-1}[$ if $N>1$. Yet, such a 
solution just belongs to $B V(\Omega)$ and satisfies the equation only in a generalized sense (see also [13] for a similar approach).

We also notice that, with the exception of [10], where bifurcation theory is used, the proofs of these results are all based on variational methods: a variant of Nehari method is used in [9], a minimization argument in [11], non-smooth critical point theory in [12]. The action functional to be considered here is

$$
\int_{\Omega}\left(\sqrt{1+\|\nabla u\|^{2}}-1\right)-\frac{\lambda}{p+1} \int_{\Omega} u^{p+1}
$$

The different behavior at zero and at infinity of the area term $\int_{\Omega}\left(\sqrt{1+\|\nabla u\|^{2}}-1\right)$, which is quadratic with respect to $\nabla u$ near the origin and grows linearly at infinity, gives raise to the multiplicity phenomena devised in the concave case; the lack of coercivity in $\mathrm{H}^{1}(\Omega)$ however causes several technical difficulties. Also, we refer the reader to paper [14] in which Obersnel and Omari, based on variational and combines critical point theory, the lower and upper solutions method and elliptic regularization, established the existence and multiplicity of positive solutions of the prescribed mean curvature problem (1.1) where $f \in \mathrm{C}(\bar{\Omega} \times \mathbb{R})$ is superlinear and do not satisfy the Ambrosetti and Rabinowitz type condition, $\lambda>0$ is a parameter. Capillarity can be briefly explained by considering the effects of two opposing forces: adhesion, i.e., the attractive (or repulsive) force between the molecules of the liquid and those of the container; and cohesion, i.e., the attractive force between the molecules of the liquid. Recently, the study of capillarity phenomena has been an interesting topic. In [15], Shokooh used variational methods to obtain existence results for elliptic equations involving the $p$-Laplacian-like. Rodrigues [16], by using Mountain Pass lemma (see [17]) and Fountain theorem (see Theorem 3.6 in [18]), established the existence of non-trivial solutions for problem

$$
\begin{cases}-\operatorname{div}\left(\left(1+\frac{|\nabla u|^{p(x)}}{\sqrt{1+|\nabla u|^{2 p(x)}}}\right)|\nabla u|^{p(x)-2} \nabla u\right)=\lambda f(x, u), & x \in \Omega, \\ u=0, & x \in \partial \Omega,\end{cases}
$$

where $\Omega \subset \mathbb{R}^{N}(N \geq 2)$ is a bounded domain with boundary of class $\mathrm{C}^{1}, \lambda$ is a positive parameter, $p \in \mathrm{C}(\bar{\Omega})$ and $f$ is a Carathéodory function. Recently, Bin [19] obtained the existence results of nontrivial solutions for every parameter $\lambda$ for the nonlinear eigenvalue problems for $p(x)$-Laplacian-like operators originated from a capillary phenomena of the following form:

$$
\begin{cases}-\operatorname{div}\left(\left(1+\frac{|\nabla u|^{p(x)}}{\sqrt{1+|\nabla u|^{2 p(x)}}}\right)|\nabla u|^{p(x)-2} \nabla u\right)=\lambda f(x, u) & \text { in } \Omega, \\ u=0 & \text { on } \partial \Omega\end{cases}
$$

where $\Omega \subset \mathbb{R}^{N}$ is a bounded domain with smooth boundary $\partial \Omega, \lambda>0$ is a parameter. Based on the mountain pass theorem a nontrivial solution is constructed for almost every parameter $\lambda>0$. he considered the continuation of the solutions. In [20], Zhou by employing variational methods, established the existence of at least one non-trivial solution for the following nonlinear eigenvalue problem for the $p(x)$-Laplacian-like operators originated from a capillary phenomenon

$$
\begin{cases}-\operatorname{div}\left(\left(1+\frac{|\nabla u|^{p(x)}}{\sqrt{1+|\nabla u|^{2 p(x)}}}\right)|\nabla u|^{p(x)-2} \nabla u\right)=\lambda f(x, u) & \text { in } \Omega, \\ u=0 & \text { on } \partial \Omega\end{cases}
$$


where $\Omega$ is a bounded domain in $\mathbb{R}^{N}$ with smooth boundary $\partial \Omega, p \in C(\bar{\Omega}), \lambda>0$ is a parameter, $f \in C(\bar{\Omega} \times \mathbb{R})$ is superlinear and does not satisfy the Ambrosetti and Rabinowitz type condition. The existence of at least one nontrivial solution was also proved.

Our approach is the variational method and the main tools are the local minimum theorem for differentiable functionals due to Bonanno [21] and Mountain Pass Theorem. Two of the consequences of the local minimum theorem due to Bonanno are here applied (see Theorems 2.1 and 2.2). Indeed, we investigate the existence of two solutions for problem $\left(P_{\lambda}^{f}\right)$ employing a consequence of the local minimum theorem due to Bonanno and mountain pass theorem under some algebraic conditions with the classical Ambrosetti-Rabinowitz (AR) condition on the nonlinear term; see [22]. Moreover, by combining two algebraic conditions on the nonlinear term applying two consequences of the local minimum theorem due to Bonanno, we guarantee the existence of two solutions. Applying the mountain pass theorem given by Pucci and Serrin ([23]), we establish the existence of third solution for problem $\left(P_{\lambda}^{f}\right)$.

The remainder of the paper is organized as follows. In Section 2, we recall the definitions and some properties of variable exponent Sobolev spaces. In Section 3, we state and prove the main results of the paper. Here, we state two special cases of our results, immediately follows from the next Theorems 3.12 and 3.17 .

Theorem 1.1. Let $p>N, \operatorname{diam}(\Omega)<\frac{N^{\frac{1}{p}}(p-N)}{p-1}$ and $h: \mathbb{R} \rightarrow \mathbb{R}$ be a non-negative continuous function such that $h(0) \neq 0$. Assume that

$$
\lim _{\xi \rightarrow 0^{+}} \frac{h(\xi)}{\xi^{p-1}}=+\infty
$$

and

(AR) there exist constants $v>p$ and $R>0$ such that, for all $|\xi| \geq R$,

$$
0<v \int_{0}^{\xi} h(\zeta) \mathrm{d} \zeta \leq \xi h(\xi)
$$

Then, for each

$$
\lambda \in\left(0, \frac{p}{2^{p-1}(1+\operatorname{meas}(\Omega))^{p}} \sup _{\gamma>0} \frac{\gamma^{p}}{\int_{0}^{\gamma} h(\zeta) \mathrm{d} \zeta}\right),
$$

where meas $(\Omega)$ is the Lebesgue measure of $\Omega$, the problem

$$
\begin{cases}-\operatorname{div}\left(\left(1+\frac{|\nabla u|^{p}}{\sqrt{1+|\nabla u|^{2 p}}}\right)|\nabla u|^{p-2} \nabla u\right)+|u|^{p-2} u=\lambda h(u(x)), & \text { in } \Omega, \\ \frac{\partial u}{\partial v}=0, & \text { on } \partial \Omega\end{cases}
$$

admits at least two positive weak solutions.

Theorem 1.2. Let $p>N$, $\operatorname{diam}(\Omega)<\frac{N^{\frac{1}{p}}(p-N)}{p-1}$ and $h: \mathbb{R} \rightarrow \mathbb{R}$ be a non-negative continuous function such that $h(0) \neq 0$. Assume that

$$
\lim _{\xi \rightarrow 0^{+}} \frac{h(\xi)}{\xi^{p-1}}=+\infty, \quad \lim _{\xi \rightarrow+\infty} \frac{h(\xi)}{\xi^{p-1}}=0
$$

and

$$
\int_{0}^{1} h(t) \mathrm{d} t<\frac{p}{2^{p}(1+\operatorname{meas}(\Omega))^{2 p}(\operatorname{meas}(\Omega))^{2}} \int_{0}^{2} h(t) \mathrm{d} t .
$$


Then for each

$$
\lambda \in\left(\frac{2^{p}(1+\operatorname{meas}(\Omega))^{p}}{\int_{0}^{2} h(t) \mathrm{d} t}, \frac{p}{(\operatorname{meas}(\Omega))^{2}(1+\operatorname{meas}(\Omega))^{p}} \frac{1}{\int_{0}^{1} h(x) \mathrm{d} x}\right),
$$

where meas $(\Omega)$ is the Lebesgue measure of $\Omega$, problem (1.3) admits at least three positive weak solutions.

\section{PRELIMINARIES}

Our basic instruments include the following theorems which derived from the existence results of a local minimum theorem for differentiable functionals due to Bonanno [21, Theorem 3.1], which is in turn motivated by Ricceri's variational principle; see $[23,24]$ and the references therein.

For a given non-empty set $X$, and two functionals $\Phi, \Psi: X \rightarrow \mathbb{R}$, we define the following functions

$$
\begin{aligned}
& \beta\left(r_{1}, r_{2}\right)=\inf _{v \in \Phi^{-1}\left(r_{1}, r_{2}\right)} \frac{\sup _{u \in \Phi^{-1}\left(r_{1}, r_{2}\right)} \Psi(u)-\Psi(v)}{r_{2}-\Phi(v)}, \\
& \rho_{1}\left(r_{1}, r_{2}\right)=\sup _{v \in \Phi^{-1}\left(r_{1}, r_{2}\right)} \frac{\Psi(v)-\sup _{u \in \Phi^{-1}\left(-\infty, r_{1}\right]} \Psi(u)}{\Phi(v)-r_{1}}
\end{aligned}
$$

for all $r_{1}, r_{2} \in \mathbb{R}, r_{1}<r_{2}$, and

$$
\rho_{2}(r)=\sup _{v \in \Phi^{-1}(r,+\infty)} \frac{\Psi(v)-\sup _{u \in \Phi^{-1}(-\infty, r]} \Psi(u)}{\Phi(v)-r}
$$

for all $r \in \mathbb{R}$.

Theorem 2.1 ([21, Theorem 5.1]). Let $X$ be a real Banach space and let $\Phi: X \rightarrow \mathbb{R}$ be a sequentially weakly lower semicontinuous, coercive and continuously Gâteaux differentiable function whose Gâteaux derivative admits a continuous inverse on $X^{*}$. Let $\Psi: X \rightarrow \mathbb{R}$ be a continuously Gâteaux differentiable function whose Gâteaux derivative is compact. Assume that there are $r_{1}, r_{2} \in \mathbb{R}, r_{1}<r_{2}$ such that

$$
\beta\left(r_{1}, r_{2}\right)<\rho_{1}\left(r_{1}, r_{2}\right) .
$$

Setting $I_{\lambda}:=\Phi-\lambda \Psi$, for each $\lambda \in\left(\frac{1}{\rho_{1}\left(r_{1}, r_{2}\right)}, \frac{1}{\beta\left(r_{1}, r_{2}\right)}\right)$, there is $u_{0, \lambda} \in \Phi^{-1}\left(r_{1}, r_{2}\right)$ such that $I_{\lambda}\left(u_{0, \lambda}\right) \leq$ $I_{\lambda}(u) \forall u \in \Phi^{-1}\left(r_{1}, r_{2}\right)$ and $I_{\lambda}^{\prime}\left(u_{0, \lambda}\right)=0$.

Theorem 2.2 ([21, Theorem 5.3]). Let $X$ be a real Banach space and let $\Phi: X \rightarrow \mathbb{R}$ be a continuously Gâteaux differentiable function whose Gâteaux derivative admits a continuous inverse on $X^{*}$. Let $\Psi$ : $X \rightarrow \mathbb{R}$ be a continuously Gâteaux differentiable function whose Gâteaux derivative is compact. Fix $\inf _{X} \Phi<r<\sup _{X} \Phi$ and assume that $\rho_{2}(r)>0$, and for each $\lambda>\frac{1}{\rho_{2}(r)}$, the functional $I_{\lambda}:=\Phi-\lambda \Psi$ is coercive. Then for each $\lambda \in\left(\frac{1}{\rho_{2}(r)},+\infty\right)$, there is $u_{0, \lambda} \in \Phi^{-1}(r,+\infty)$ such that $I_{\lambda}\left(u_{0, \lambda}\right) \leq I_{\lambda}(u) \forall u \in$ $\Phi^{-1}(r,+\infty)$ and $I_{\lambda}^{\prime}\left(u_{0, \lambda}\right)=0$.

We refer readers to the paper [25] in which Theorems 2.1 and 2.2 have been successfully employed to obtain the existence of solutions for nonlinear mixed boundary value problems Here and in the sequel, meas $(\Omega)$ denotes the Lebesgue measure of the set $\Omega$, and we also assume that $p \in \mathrm{C}_{+}(\Omega):=\{h \in \mathrm{C}(\bar{\Omega})$ : $\mathrm{h}(\mathrm{x})>1, \forall \mathrm{x} \in \bar{\Omega}\}$ verifies the following condition:

$$
N<p^{-}:=\inf _{x \in \Omega} p(x) \leq p(x) \leq p^{+}:=\sup _{x \in \Omega} p(x)<+\infty .
$$


Define the variable exponent Lebesgue space by

$$
\mathrm{L}^{p(x)}(\Omega):=\left\{u: \Omega \rightarrow \mathbb{R} \text { measurable and } \int_{\Omega}|u(x)|^{p(x)} \mathrm{d} x<+\infty\right\} .
$$

On $\mathrm{L}^{p(x)}(\Omega)$ we consider the norms respectively

$$
\|u\|_{\mathrm{L}^{p(x)}(\Omega)}=\inf \left\{\eta>0: \int_{\Omega}\left|\frac{u(x)}{\eta}\right|^{p(x)} \mathrm{d} x \leq 1\right\} .
$$

Let $X$ be the generalized Lebesgue-Sobolev space $\mathrm{W}^{1, p(x)}(\Omega)$ defined by putting $\mathrm{W}^{1, p(x)}(\Omega)$ by

$$
\mathrm{W}^{1, p(x)}(\Omega)=\left\{u \in \mathrm{L}^{p(x)}(\Omega):|\nabla u| \in \mathrm{L}^{p(x)}(\Omega)\right\}
$$

endowed with the following norm

$$
\|u\|_{\mathrm{W}^{1, p(x)}(\Omega)}:=\|u\|_{\mathrm{L}^{p(x)}(\Omega)}+\|\mid \nabla u\|_{\mathrm{L}^{p(x)}(\Omega)} .
$$

It is well known (see [26]) that, in view of (2.1), both $\mathrm{L}^{p(x)}(\Omega)$ and $\mathrm{W}^{1, p(x)}(\Omega)$, with the respective norms, are separable, reflexive and uniformly convex Banach spaces. Moreover, since $\alpha \in \mathrm{L}^{\infty}(\Omega)$, with $\alpha^{-}:=\operatorname{essinf}_{x \in \Omega} \alpha(x)>0$ is assumed, the norm

$$
\|u\|_{\alpha}=\inf \left\{\sigma>0: \int_{\Omega}\left(\alpha(x)\left|\frac{u(x)}{\sigma}\right|^{p(x)}+\left|\frac{\nabla u(x)}{\sigma}\right|^{p(x)}\right) \mathrm{d} x \leq 1\right\}
$$

on $\mathrm{W}^{1, p(x)}(\Omega)$ is equivalent to that introduce in (2.2). Since $\mathrm{W}^{1, p(x)}(\Omega)$ is continuously embedded in $\mathrm{W}^{1, p^{-}}(\Omega)$ (see [26] or [27]) and $p^{-}>N, \mathrm{~W}^{1, p(x)}(\Omega)$ is continuously embedded in $\mathrm{C}^{0}(\bar{\Omega})$ and one has

$$
\|u\|_{\mathrm{C}^{0}(\bar{\Omega})} \leq k_{p^{-}}\|u\|_{\mathrm{W}^{1, p^{-}}(\Omega)} .
$$

When $\Omega$ is convex, an explicit upper bound for the constant $k_{p^{-}}$is

$$
k_{p^{-}} \leq 2^{\frac{p^{-}-1}{p^{-}}} \max \left\{\left(\frac{1}{\|\alpha\|_{\mathrm{L}^{1}(\Omega)}}\right)^{\frac{1}{p^{-}}}, \frac{D}{N^{\frac{1}{p^{-}}}}\left(\frac{p^{-}-1}{p^{-}-N} \operatorname{meas}(\Omega)\right)^{\frac{p^{-}-1}{p^{-}}} \frac{\|\alpha\|_{\infty}}{\|\alpha\|_{\mathrm{L}^{1}(\Omega)}}\right\},
$$

where $D=\operatorname{diam}(\Omega)$ and meas $(\Omega)$ is the Lebesgue measure of $\Omega$ (see [28, Remark 1]). On the other hand, taking into account that $p^{-} \leq p(x),\left[27\right.$, Theorem 2.8] ensures that $\mathrm{L}^{p(x)}(\Omega) \hookrightarrow \mathrm{L}^{p^{-}}(\Omega)$ and the constant of such embedding does not exceed $1+$ meas $(\Omega)$. So, one has

$$
\|u\|_{\mathrm{W}^{1, p^{-}}(\Omega)} \leq(1+\operatorname{meas}(\Omega))\|u\|_{\mathrm{W}^{1, p(x)}(\Omega)} \leq(1+\operatorname{meas}(\Omega))\|\alpha\|_{\mathrm{L}^{1}(\Omega)} .
$$

In conclusion, putting

$$
\rho=k_{p^{-}}(1+\operatorname{meas}(\Omega)),
$$

it results

$$
\|u\|_{C^{0}(\bar{\Omega})} \leq \rho\|u\|_{\alpha}
$$

for each $u \in \mathrm{W}^{1, p(x)}(\Omega)$. Put

$$
F(x, t):=\int_{0}^{t} f(t, \xi) \mathrm{d} \xi \quad \text { for all }(t, x) \in \Omega \times \mathbb{R} .
$$


Definition 2.3. We mean by a (weak) solution of problem $\left(P_{\lambda}^{f}\right)$, any function $u \in X$ such that

$$
\begin{aligned}
& \int_{\Omega}\left[\left(|\nabla u(x)|^{p(x)-2} \nabla u(x)+\frac{|\nabla u(x)|^{2 p(x)-2} \nabla u(x)}{\sqrt{1+|\nabla u(x)|^{2 p(x)}}}\right) \nabla v(x)\right. \\
& \left.+\alpha(x)|u(x)|^{p(x)-2} u(x) v(x)\right] \mathrm{d} x-\lambda \int_{\Omega} f(x, u(x)) v(x) \mathrm{d} x=0
\end{aligned}
$$

for every $v \in \mathrm{W}^{1, p(x)}(\Omega)$.

Proposition 2.4 ([2, Proposition 2.4]). Letting $\rho_{\alpha}(u)=\int_{\Omega}\left(|\nabla u|^{p(x)}+\alpha(x)|u|^{p(x)}\right) \mathrm{d} x$ for $u \in \mathrm{W}^{1, p(x)}(\Omega)$, we have

(1) $\|u\|_{\alpha} \geq 1 \Longrightarrow\|u\|_{\alpha}^{p^{-}} \leq \rho_{\alpha}(u) \leq\|u\|_{\alpha}^{p^{+}}$,

(2) $\|u\|_{\alpha} \leq 1 \Longrightarrow\|u\|_{\alpha}^{p^{+}} \leq \rho_{\alpha}(u) \leq\|u\|_{\alpha}^{p^{-}}$.

Now for every $u \in X$, we define

$$
\Phi(u):=\int_{\Omega} \frac{1}{p(x)}\left(|\nabla u(x)|^{p(x)}+\sqrt{1+|\nabla u(x)|^{2 p(x)}}+\alpha(x)|u(x)|^{p(x)}\right) \mathrm{d} x,
$$

and

$$
\Psi(u)=\int_{\Omega} F(x, u(x)) \mathrm{d} x .
$$

Standard arguments show that $\Phi$ and $\Psi$ are Gâteaux differentiable functionals whose Gâteaux derivatives at the point $u \in X$ are given by

$$
\begin{aligned}
\Phi^{\prime}(u)(v)= & \int_{\Omega}\left[\left(|\nabla u(x)|^{p(x)-2} \nabla u(x)+\frac{|\nabla u(x)|^{2 p(x)-2} \nabla u(x)}{\sqrt{1+|\nabla u(x)|^{2 p(x)}}}\right) \nabla v(x)\right. \\
& \left.+\alpha(x)|u(x)|^{p(x)-2} u(x) v(x)\right] \mathrm{d} x
\end{aligned}
$$

and

$$
\Psi^{\prime}(u)(v)=\int_{\Omega} f(x, u(x)) v(x) \mathrm{d} x
$$

for all $u, v \in X$, respectively. Hence, a critical point of the functional $\Phi-\lambda \Psi$, gives us a weak solution of $\left(P_{\lambda}^{f}\right)$.

We need the following proposition in the proofs of our main results.

Proposition 2.5 ([16]). The functional $\varphi: X \rightarrow \mathbb{R}$ defined by

$$
\varphi(u):=\int_{\Omega} \frac{1}{p(x)}\left(|\nabla u(x)|^{p(x)}+\sqrt{1+|\nabla u(x)|^{2 p(x)}}\right) \mathrm{d} x
$$

is convex and the mapping $\varphi^{\prime}: X \rightarrow X^{*}$ is a strictly monotone and bounded homeomorphism.

Remark 2.6. We say that $f: \Omega \times \mathbb{R} \rightarrow \mathbb{R}$ is an $\mathrm{L}^{1}$-Carathéodory function, if

(a) $x \mapsto f(x, t)$ is measurable for every $t \in \mathbb{R}$,

(b) $t \mapsto f(x, t)$ is continuous for a.e. $x \in \Omega$,

(c) for every $\rho>0$ there exists a function $l_{\rho} \in L^{1}(\Omega)$ such that

$$
\sup _{|t| \leq \rho}|f(x, t)| \leq l_{\rho}(x)
$$

for a.e. $x \in \Omega$. 


\section{MAIN RESULTS}

In this section we formulate our main results. For a non-negative constant $\gamma$ and a positive constant $\delta$ with $\gamma^{p^{-}} \neq \delta^{p^{+}}\|\alpha\|_{\mathrm{L}^{1}(\Omega)}$, we set

$$
a_{\gamma}(\delta):=\frac{\int_{\Omega} \sup _{|t| \leq \gamma} F(x, t) \mathrm{d} x-\int_{\Omega} F(x, \delta) \mathrm{d} x}{\gamma^{p^{-}}-\rho^{p^{-}} \delta^{p^{+}}\|\alpha\|_{L^{1}(\Omega)}} .
$$

Theorem 3.1. Assume that $f(x, 0) \neq 0$ for all $x \in \Omega$ and suppose that there exist a non-negative constant $\gamma_{1}$ and two positive constants $\gamma_{2}$ and $\delta$, with

$$
\frac{\gamma_{1}}{\rho\|\alpha\|_{\mathrm{L}^{1}(\Omega)}}<\delta<\sqrt[p^{+}]{\frac{p^{-}}{p^{+}\|\alpha\|_{\mathrm{L}^{1}(\Omega)} \rho^{p^{-}}}} \gamma_{2}^{p^{p^{+}}}
$$

such that

$\left(\mathrm{A}_{1}\right) \quad a_{\gamma_{2}}(\boldsymbol{\delta})<a_{\gamma_{1}}(\boldsymbol{\delta})$

$\left(\mathrm{A}_{2}\right) \quad$ there exist $v>p^{+}$and $R>0$ such that

$$
0<v F(x, \xi) \leq \xi f(x, \xi)
$$

for all $|\xi| \geq R$ and for all $x \in \Omega$.

Then, for each

$$
\lambda \in\left(\frac{p^{+}}{\rho^{p^{-}}} \frac{1}{a_{\gamma_{1}}(\boldsymbol{\delta})}, \frac{p^{+}}{\rho^{p^{-}}} \frac{1}{a_{\gamma_{2}}(\boldsymbol{\delta})}\right)
$$

problem $\left(P_{\lambda}^{f}\right)$ admits at least two non-trivial weak solutions $u_{1}$ and $u_{2}$ in $X$, such that

$$
\begin{aligned}
\frac{1}{p^{+}}\left(\frac{\gamma_{1}}{\rho}\right)^{p^{-}} & \leq \int_{\Omega} \frac{1}{p(x)}\left(\left|\nabla u_{1}(x)\right|^{p(x)}+\sqrt{1+\left|\nabla u_{1}(x)\right|^{2 p(x)}}+\alpha(x)\left|u_{1}(x)\right|^{p(x)}\right) \mathrm{d} x \\
& \leq \frac{1}{p^{+}}\left(\frac{\gamma_{2}}{\rho}\right)^{p^{-}} .
\end{aligned}
$$

Proof. Put $I_{\lambda}=\Phi-\lambda \Psi$, where $\Phi$ and $\Psi$ are given as in (2.4) and (2.5), respectively. Due to Proposition 2.5 , we have

$$
\Phi(u) \geq \frac{1}{p^{+}}\|u\|_{\alpha}^{p^{-}}
$$

for all $u \in X$ with $\|u\|>1$, which follows that $\Phi$ is coercive. Of course $\Phi$ is sequentially weakly lower semicontinuous and continuously Gâteaux differentiable while Proposition 2.5 gives that its Gâteaux derivative admits a continuous inverse on $X^{*}$. The functional $\Psi: X \rightarrow \mathbb{R}$ is well defined and is continuously Gâteaux differentiable whose Gâteaux derivative is compact. Choose

$$
r_{1}=\frac{1}{p^{+}}\left(\frac{\gamma_{1}}{\rho}\right)^{p^{-}}, \quad r_{2}=\frac{1}{p^{+}}\left(\frac{\gamma_{2}}{\rho}\right)^{p^{-}}
$$

and for $\delta>1$ define $w_{\delta} \in X$ by

$$
w_{\delta}(t)=\delta
$$

Thus

$$
\begin{aligned}
\Phi\left(w_{\delta}\right) & =\int_{\Omega} \frac{1}{p(x)}\left(\left|\nabla w_{\delta}(x)\right|^{p(x)}+\sqrt{1+\left|\nabla w_{\delta}(x)\right|^{2 p(x)}}+\alpha(x)\left|w_{\delta}(x)\right|^{p(x)}\right) \mathrm{d} x \\
& =\int_{\Omega} \frac{1}{p(x)}\left(\alpha(x)|\delta|^{p(x)}\right) \mathrm{d} x
\end{aligned}
$$


According to (2.1), we have the following inequalities

$$
\frac{\delta^{p^{-}}}{p^{+}}\|\alpha\|_{\mathrm{L}^{1}(\Omega)} \leq \Phi\left(w_{\delta}\right) \leq \frac{\delta^{p^{+}}}{p^{-}}\|\alpha\|_{\mathrm{L}^{1}(\Omega)} .
$$

Moreover, for all $u \in X$ with $\Phi(u)<r_{1}$, one has

$$
\|u\|_{\alpha} \leq \max \left\{\left(p^{+} r_{1}\right)^{\frac{1}{p^{+}}},\left(p^{+} r_{1}\right)^{\frac{1}{p^{1}}}\right\}=\frac{\gamma_{1}}{\rho} .
$$

So, due to the embedding $X \hookrightarrow \mathrm{C}^{0}(\Omega)$ (see (2.3)), one has $\|u\|_{\infty} \leq \rho\|u\|_{\alpha}<\gamma_{1}$. It follows that

$$
\Phi^{-1}\left(-\infty, r_{1}\right)=\left\{u \in X ; \Phi(u)<r_{1}\right\} \subseteq\left\{u \in X ;|u| \leq \gamma_{1}\right\},
$$

which concludes

$$
\Psi(u) \leq \sup _{u \in \Phi^{-1}\left(-\infty, r_{1}\right)} \int_{\Omega} F(x, u(x)) \mathrm{d} x \leq \int_{\Omega} \sup _{|t| \leq \gamma_{1}} F(x, t) \mathrm{d} x .
$$

By the same argument as above we have

$$
\sup _{u \in \Phi^{-1}\left(-\infty, r_{2}\right)} \Psi(u) \leq \int_{\Omega} \sup _{|t| \leq \gamma_{2}} F(x, t) \mathrm{d} x .
$$

Therefore,

$$
\begin{aligned}
\beta\left(r_{1}, r_{2}\right) & \leq \frac{\sup _{u \in \Phi^{-1}\left(-\infty, r_{2}\right)} \Psi(u)-\Psi\left(w_{\delta}\right)}{r_{2}-\Phi\left(w_{\delta}\right)} \\
& \leq \frac{p^{+}}{\rho^{p^{-}}} \frac{\int_{\Omega} \sup _{|t| \leq \gamma_{2}} F(x, t) \mathrm{d} x-\int_{\Omega} F(x, \delta) \mathrm{d} x}{\gamma_{2}^{p^{-}}-\rho^{p^{-}} \delta^{p^{+}}\|\alpha\|_{\mathrm{L}^{1}(\Omega)}} \\
& =\frac{p^{+}}{\rho^{p^{-}}} a_{\gamma_{2}}(\delta) .
\end{aligned}
$$

On the other hand, one has

$$
\begin{aligned}
\rho_{2}\left(r_{1}, r_{2}\right) & \geq \frac{\Psi\left(w_{\delta}\right)-\sup _{u \in \Phi^{-1}\left(-\infty, r_{1}\right]} \Psi(u)}{\Phi\left(w_{\delta}\right)-r_{1}} \\
& \geq \frac{p^{+}}{\rho^{p^{-}}} \frac{\int_{\Omega} \sup _{|t| \leq \gamma_{1}} F(x, t) \mathrm{d} x-\int_{\Omega} F(x, \delta) \mathrm{d} x}{\gamma_{1}^{p^{-}}-\rho^{p^{-}} \delta^{p^{+}}\|\alpha\|_{\mathrm{L}^{1}(\Omega)}} \\
& =\frac{p^{+}}{\rho^{p^{-}}} a_{\gamma_{1}}(\delta) .
\end{aligned}
$$

Hence, from $\left(A_{1}\right)$, one has $\beta\left(r_{1}, r_{2}\right)<\rho_{2}\left(r_{1}, r_{2}\right)$. Therefore, from Theorem 2.1, for each

$$
\lambda \in\left(\frac{p^{+}}{\rho^{p^{-}}} \frac{1}{a_{\gamma_{1}}(\delta)}, \frac{p^{+}}{\rho^{p^{-}}} \frac{1}{a_{\gamma_{2}}(\delta)}\right),
$$

the functional $I_{\lambda}$ admits at least one non-trivial critical point $u_{1}$ such that

$$
r_{1}<\Phi\left(u_{1}\right)<r_{2}
$$

that is,

$$
\begin{aligned}
\frac{1}{p^{+}}\left(\frac{\gamma_{1}}{\rho}\right)^{p^{-}} & \leq \int_{\Omega} \frac{1}{p(x)}\left(\left|\nabla u_{1}(x)\right|^{p(x)}+\sqrt{1+\left|\nabla u_{1}(x)\right|^{2 p(x)}}+\alpha(x)\left|u_{1}(x)\right|^{p(x)}\right) \mathrm{d} x \\
& \leq \frac{1}{p^{+}}\left(\frac{\gamma_{2}}{\rho}\right)^{p^{-}} .
\end{aligned}
$$


Now, we prove the existence of the second local minimum distinct from the first one. To this aim, we verify the hypotheses of the mountain-pass theorem for the functional $I_{\lambda}$. Clearly, the functional $I_{\lambda}$ is of class $C^{1}$ and $I_{\lambda}(0)=0$. The first part of proof guarantees that $u_{1} \in X$ is a local nontrivial local minimum for $I_{\lambda}$ in $X$. We can assume that $u_{1}$ is a strict local minimum for $I_{\lambda}$ in $X$. Therefore, there is $\rho^{*}>0$ such that $\inf _{\left\|u-u_{1}\right\|=\rho^{*}} I_{\lambda}(u)>I_{\lambda}\left(u_{1}\right)$, so condition [29, $\left(I_{1}\right)$, Theorem 2.2] is verified. By integrating the condition (3.2), there exist constants $a_{1}, a_{2}>0$ such that

$$
F(x, t) \geq a_{1}|t|^{v}-a_{2}
$$

for all $x \in \Omega$ and $t \in \mathbb{R}$. Now, choosing any $u \in X \backslash\{0\}$ and taking Proposition 2.4 into account, one has

$$
\begin{aligned}
I_{\lambda}(\tau u) & =(\Phi-\lambda \Psi)(\tau u) \\
& \leq \frac{1}{p^{-}} \max \left\{\|\tau u\|^{p^{+}},\|\tau u\|^{p^{-}}\right\}-\lambda \int_{\Omega} F(x, \tau u(x)) \mathrm{d} x \\
& \leq \frac{\max \left\{\tau^{p^{+}}, \tau^{p^{-}}\right\}}{p^{-}} \max \left\{\|u\|^{p^{+}},\|u\|^{p^{-}}\right\}-\lambda \tau^{v} a_{1} \int_{\Omega}|u(x)|^{v} \mathrm{~d} x+\lambda a_{2} \\
& \rightarrow-\infty
\end{aligned}
$$

as $\tau \rightarrow+\infty$, so condition [29, $\left(I_{2}\right)$, Theorem 2.2] is satisfied. Thus, the functional $I_{\lambda}$ satisfies the geometry of mountain pass. Moreover, $I_{\lambda}$ satisfies the Palais-Smale condition. Indeed, assume that $\left\{u_{n}\right\}_{n \in \mathbb{N}} \subset X$ such that $\left\{I_{\lambda}\left(u_{n}\right)\right\}_{n \in \mathbb{N}}$ is bounded and $I_{\lambda}^{\prime}\left(u_{n}\right) \rightarrow 0$ as $n \rightarrow+\infty$. Then, there exists a positive constant $c_{0}$ such that

$$
\left|I_{\lambda}\left(u_{n}\right)\right| \leq c_{0},\left|I_{\lambda}^{\prime}\left(u_{n}\right)\right| \leq c_{0} \forall n \in \mathbb{N} .
$$

Therefore, we infer to deduce from the definition of $I_{\lambda}^{\prime}$ and the assumption $\left(A_{2}\right)$ that

$$
\begin{aligned}
c_{0}+c_{1}\left\|u_{n}\right\| \geq & v I_{\lambda}\left(u_{n}\right)-I_{\lambda}^{\prime}\left(u_{n}\right)\left(u_{n}\right) \geq\left(\frac{v}{p^{+}}-1\right) \min \left\{\left\|u_{n}\right\|_{\alpha}^{p^{+}},\left\|u_{n}\right\|_{\alpha}^{p^{-}}\right\} \\
& -\lambda \int_{\Omega}\left(v F\left(x, u_{n}(x)\right)-f\left(x, u_{n}(x)\right)\left(u_{n}(x)\right)\right) \mathrm{d} x \\
\geq & \left(\frac{v}{p^{+}}-1\right) \min \left\{\left\|u_{n}\right\|_{\alpha}^{p^{+}},\left\|u_{n}\right\|_{\alpha}^{p^{-}}\right\}
\end{aligned}
$$

for some $c_{1}>0$. Since $v>p^{+}$, this implies that $\left(u_{n}\right)$ is bounded. Consequently, since $X$ is a reflexive Banach space we have, up to a subsequence,

$$
u_{n} \rightarrow u \quad \text { in } X
$$

By $I_{\lambda}^{\prime}\left(u_{n}\right) \rightarrow 0$ and $u_{n} \rightarrow u$ in $X$, we obtain

$$
\left(I_{\lambda}^{\prime}\left(u_{n}\right)-I_{\lambda}^{\prime}(u)\right)\left(u_{n}-u\right) \rightarrow 0
$$

From the continuity of $f$, we have

$$
\int_{\Omega}\left(f\left(x, u_{n}(x)\right)-f(x, u(x))\right)\left(u_{n}(x)-u(x)\right) \mathrm{d} x \rightarrow 0, \quad \text { as } n \rightarrow+\infty .
$$

So by (3.6) and [16, Proposition 3.1] the sequence $\left\{u_{n}\right\}$ converges strongly to $u$ in $X$. Therefore, $I_{\lambda}$ satisfies the Palais-Smale condition. Hence, by the classical theorem of Ambrosetti and Rabinowitz we establish a critical point $u_{2}$ of $I_{\lambda}$ such that $I_{\lambda}\left(u_{2}\right)>I_{\lambda}\left(u_{1}\right)$. Since $f(x, 0) \neq 0$ for all $x \in \Omega, u_{1}$ and $u_{2}$ are two distinct nontrivial weak solutions of $\left(P_{\lambda}^{f}\right)$ and the proof is complete. 
Remark 3.2. In Theorem 3.1, we guaranteed the existence of at least two nontrivial weak solutions for $\left(P_{\lambda}^{f}\right)$. One of these solutions has been achieved in relation with the classical Ambrosetti-Rabinowitz condition on the data by taking $f(x, 0) \neq 0$ for all $x \in \Omega$. If $f(x, 0) \neq 0$ for all $x \in \Omega$ does not hold, the second solution $u_{2}$ of the problem $\left(P_{\lambda}^{f}\right)$ may be trivial, but the problem has at least a nontrivial solution.

Remark 3.3. In Theorem 3.1, we looked for the critical points of the functional $I_{\lambda}$ naturally associated with problem $\left(P_{\lambda}^{f}\right)$. We note that, in general, $I_{\lambda}$ can be unbounded from the following in $X$. Indeed, for example, in the case when $f(x, \xi)=1+|\xi|^{a-p^{+}} \xi p^{p^{+}-1}$ for $(x, \xi) \in \Omega \times \mathbb{R}$ with $a>p^{+}$, for any fixed $u \in X \backslash\{0\}$ and $\imath \in \mathbb{R}$, we obtain

$$
\begin{aligned}
& I_{\lambda}(\imath u)=\Phi(\imath u)-\lambda \int_{\Omega} F(x, \imath u(x)) \mathrm{d} x \\
& \leq \frac{\imath^{p^{+}}}{p^{-}}\|u\|^{p^{+}}-\lambda \imath\|u\|_{\mathrm{L}^{1}(\Omega)}-\lambda \frac{\imath^{a}}{a}\|u\|_{\mathrm{L}^{a}(\Omega)}^{a} \rightarrow-\infty
\end{aligned}
$$

as $\imath \rightarrow+\infty$. Hence, we can not use direct minimization to find critical points of the functional $I_{\lambda}$.

Now, we point out an immediate consequence of Theorem 3.1.

Theorem 3.4. Assume that $f(x, 0) \neq 0$ for all $x \in \Omega$ and there exist two positive constants $\delta$ and $\gamma$, with

$$
\delta<\sqrt[p^{+}]{\frac{p^{-}}{p^{+}\|\alpha\|_{L^{1}(\Omega)} \rho^{p^{-}}}} \gamma^{\frac{p^{-}}{p^{+}}}
$$

such that the assumption $\left(A_{2}\right)$ in Theorem 3.1 holds. Furthermore, suppose that

$$
\frac{\int_{\Omega} \sup _{|t| \leq \gamma} F(x, t) \mathrm{d} x}{\gamma^{p^{-}}}<\frac{1}{\rho^{p^{-}}\|\alpha\|_{\mathrm{L}^{1}(\Omega)}} \frac{\int_{\Omega} F(x, \delta) \mathrm{d} x}{\delta^{p^{+}}} .
$$

Then, for each

$$
\lambda \in\left(\frac{p^{+} \delta^{p^{+}}\|\alpha\|_{\mathrm{L}^{1}(\Omega)}}{\int_{\Omega} F(x, \delta) \mathrm{d} x}, \frac{p^{+} \gamma^{p^{-}}}{\rho^{p^{-}} \int_{\Omega} \sup _{|t| \leq \gamma} F(x, t) \mathrm{d} x}\right),
$$

problem $\left(P_{\lambda}^{f}\right)$ admits at least two non-trivial weak solutions $u_{1}$ and $u_{2}$ in $X$ such that

$$
\begin{aligned}
& 0<\int_{\Omega} \frac{1}{p(x)}\left(\left|\nabla u_{1}(x)\right|^{p(x)}+\sqrt{1+\left|\nabla u_{1}(x)\right|^{2 p(x)}}+\alpha(x)\left|u_{1}(x)\right|^{p(x)}\right) \mathrm{d} x \\
& \leq \frac{1}{p^{+}}\left(\frac{\gamma}{\rho}\right)^{p^{-}} .
\end{aligned}
$$

Proof. The conclusion follows from Theorem 3.1, by taking $\gamma_{1}=0$ and $\gamma_{2}=\gamma$. Indeed, owing to the inequality (3.8) and $\left(A_{1}\right)$, one has

$$
\begin{aligned}
a_{\gamma}(\delta) & =\frac{\int_{\Omega} \sup _{|t| \leq \gamma} F(x, t) \mathrm{d} x-\int_{\Omega} F(x, \delta) \mathrm{d} x}{\gamma^{p^{-}}-\rho^{p^{-}} \delta^{p^{+}}\|\alpha\|_{\mathrm{L}^{1}(\Omega)}} \\
& <\frac{\int_{\Omega} \sup _{|t| \leq \gamma} F(x, t) \mathrm{d} x}{\gamma^{p^{-}}} \\
& <\frac{\int_{\Omega} F(x, \delta) \mathrm{d} x}{\rho^{p^{-}} \delta p^{+}\|\alpha\|_{\mathrm{L}^{1}(\Omega)}} \\
& =a_{0}(\boldsymbol{\delta}) .
\end{aligned}
$$


In particular, one has

which follows

$$
a_{\gamma}(\delta)<\frac{\int_{\Omega} \sup _{|t| \leq \gamma} F(x, t) \mathrm{d} x}{\gamma^{p^{-}}}
$$

$$
\frac{p^{+}}{\rho^{p^{-}}} \frac{\gamma^{p^{-}}}{\int_{\Omega} \sup _{|t| \leq \gamma} F(x, t) \mathrm{d} x}<\frac{p^{+}}{\rho^{p^{-}}} \frac{1}{a_{\gamma}(\boldsymbol{\delta})} .
$$

Hence, Theorem 3.1 ensures the conclusion.

Now we illustrate Theorem 3.4 by presenting the following example.

Example 3.5. Let $N=1, \Omega=\left(0, \frac{\pi}{2}\right) \subset \mathbb{R}, p(x)=3+2 \sin x$ for all $x \in\left[0, \frac{\pi}{2}\right], \alpha(x)=x^{9}$ for all $x \in\left[0, \frac{\pi}{2}\right]$ and

$$
f(x, t)= \begin{cases}e^{x}\left(1+t^{8}\right), & |t| \geq 1, x \in\left(0, \frac{\pi}{2}\right), \\ e^{x}\left(3-t^{2}\right), & |t|<1, x \in\left(0, \frac{\pi}{2}\right) .\end{cases}
$$

Thus, $p^{-}=3, p^{+}=4$, meas $(\Omega)=\frac{\pi}{2}, D=\frac{\pi}{2},\|\alpha\|_{\mathrm{L}^{1}(\Omega)}=\frac{1}{10}\left(\frac{\pi}{2}\right)^{10},\|\alpha\|_{\infty}=\left(\frac{\pi}{2}\right)^{9}$ and $k_{p^{-}}=k_{3} \leq$ $\sqrt[3]{40\left(\frac{2}{\pi}\right)^{10}}$, and so, $\rho \leq \sqrt[3]{40\left(\frac{2}{\pi}\right)^{10}}\left(1+\frac{\pi}{2}\right)$. Moreover, $f$ is an $\mathrm{L}^{1}$-Carathéodory and non-negative function, $f(x, 0) \neq 0$ for all $x \in\left(0, \frac{\pi}{2}\right)$ and

$$
F(x, t)= \begin{cases}e^{x}\left(t+\frac{t^{9}}{9}-\frac{14}{9}\right), & t<-1, x \in\left(0, \frac{\pi}{2}\right) \\ e^{x}\left(3 t-\frac{t^{3}}{3}\right), & -1 \leq t \leq 1, x \in\left(0, \frac{\pi}{2}\right), . \\ e^{x}\left(t+\frac{t^{9}}{9}+\frac{14}{9}\right), & t>1, x \in\left(0, \frac{\pi}{2}\right) .\end{cases}
$$

Now, since

$$
\lim _{|\xi| \rightarrow+\infty} \frac{\xi f(x, \xi)}{F(x, \xi)}=\lim _{|\xi| \rightarrow+\infty} \frac{e^{x}\left(t+t^{9}\right)}{t+\frac{t^{9}}{9}}=9 e^{x} \geq 9>4=p^{+}
$$

for all $x \in\left(0, \frac{\pi}{2}\right)$, by choosing $v=\frac{17}{2}>4=p^{+}$we can choose $R>1$ in a manner that the assumption (3.2) is fulfilled. Moreover, by choosing $\delta=\frac{1}{4}$ and $\gamma=1$ the assumptions (3.7) and (3.8) are fulfilled. Indeed,

$$
\delta=\frac{1}{4}<\frac{1}{2} \sqrt[4]{\frac{3}{\left(1+\frac{\pi}{2}\right)^{3}}} \leq \sqrt[p^{+}]{\frac{p^{-}}{p^{+}\|\alpha\|_{L^{1}(\Omega)} \rho^{p^{-}}}} \gamma^{p^{p^{-}}}
$$

and

$$
\begin{aligned}
& \frac{\int_{\Omega} \sup _{|t| \leq \gamma} F(x, t) \mathrm{d} x}{\gamma^{p^{-}}}=\frac{8}{3}\left(e^{\frac{\pi}{2}}-1\right) \\
& <\frac{143}{3\left(1+\frac{\pi}{2}\right)^{3}}\left(e^{\frac{\pi}{2}}-1\right) \leq \frac{1}{\rho^{p^{-}}\|\alpha\|_{\mathrm{L}^{1}(\Omega)}} \frac{\int_{\Omega} F(x, \delta) \mathrm{d} x}{\delta^{p^{+}}} .
\end{aligned}
$$

Therefore, by apply Theorem 3.4 for each

$$
\lambda \in\left(\frac{3}{1430\left(e^{\frac{\pi}{2}}-1\right)}\left(\frac{\pi}{2}\right)^{10}, \frac{3}{10(2+\pi)\left(e^{\frac{\pi}{2}}-1\right)}\left(\frac{\pi}{2}\right)^{10}\right)
$$

the problem

$$
\left\{\begin{array}{l}
-\left(\left(1+\frac{\left|u^{\prime}(x)\right|^{3+2 \sin x}}{\sqrt{1+\left|u^{\prime}\right|^{6+4 \sin x}}}\right)\left|u^{\prime}(x)\right|^{1+2 \sin x} u^{\prime}(x)\right)^{\prime}=\lambda f(x, t), \quad \text { in }\left(0, \frac{\pi}{2}\right), \\
u^{\prime}(0)=u^{\prime}\left(\frac{\pi}{2}\right)=0
\end{array}\right.
$$


admits at least two non-trivial weak solutions $u_{1}$ and $u_{2}$ in the space

$$
\mathrm{W}^{1,3+2 \sin x}\left(0, \frac{\pi}{2}\right)=\left\{u \in \mathrm{L}^{3+2 \sin x}\left(0, \frac{\pi}{2}\right): u^{\prime} \in \mathrm{L}^{3+2 \sin x}\left(0, \frac{\pi}{2}\right)\right\}
$$

such that

$$
\begin{aligned}
& 0<\int_{0}^{\frac{\pi}{2}} \frac{1}{3+2 \sin x}\left(\left|u_{1}^{\prime}(x)\right|^{3+2 \sin x}+\sqrt{1+\left|u_{1}^{\prime}(x)\right|^{6+4 \sin x}}+x^{9}\left|u_{1}(x)\right|^{3+2 \sin x}\right) \mathrm{d} x \\
& \leq \frac{1}{20(\pi+2)^{3}}\left(\frac{\pi}{2}\right)^{10} .
\end{aligned}
$$

Now, we give an application of Theorem 2.2 which will be used later to obtain multiple solutions for $\operatorname{problem}\left(P_{\lambda}^{f}\right)$.

Theorem 3.6. Assume that there exist two positive constants $\bar{\gamma}$ and $\bar{\delta}$ with

$$
\bar{\delta}<\sqrt[p^{+}]{\frac{p^{-}}{p^{+}\|\alpha\|_{L^{1}(\Omega)} \rho^{p^{-}}}} \bar{\gamma}_{2}^{p^{+}}
$$

such that

$$
\int_{\Omega} \sup _{|t| \leq \bar{\gamma}} F(x, t) \mathrm{d} x<\int_{\Omega} F(x, \bar{\delta}) \mathrm{d} x
$$

and

$$
\limsup _{|\xi| \rightarrow+\infty} \frac{F(x, \xi)}{|\xi|^{p^{-}}} \leq 0 \quad \text { uniformly in } \mathbb{R}
$$

Then, for each $\lambda>\tilde{\lambda}$, where

$$
\tilde{\lambda}:=\frac{p^{+}}{\rho^{p^{-}}} \frac{\rho^{p^{-}} \delta^{p^{+}}\|\alpha\|_{\mathrm{L}^{1}(\Omega)}-\bar{\gamma}^{p^{-}}}{\int_{\Omega} F(x, \bar{\delta}) \mathrm{d} x-\int_{\Omega} \sup _{|t| \leq \bar{\gamma}} F(x, t) \mathrm{d} x},
$$

problem $\left(P_{\lambda}^{f}\right)$ admits at least one non-trivial weak solution $\overline{u_{1}} \in X$ such that

$$
\int_{\Omega} \frac{1}{p(x)}\left(\left|\nabla \overline{u_{1}}(x)\right|^{p(x)}+\sqrt{1+\left|\nabla \overline{u_{1}}(x)\right|^{2 p(x)}}+\alpha(x)\left|\overline{u_{1}}(x)\right|^{p(x)}\right) \mathrm{d} x>\frac{1}{p^{+}}\left(\frac{\bar{\gamma}}{\rho}\right)^{p^{-}} .
$$

Proof. Take the real Banach space $X$ as defined in Section 2, and put $I_{\lambda}=\Phi-\lambda \Psi$, where $\Phi$ and $\Psi$ are given as in (2.4) and (2.5), respectively. Our goal is to apply Theorem 2.2 to function $I_{\lambda}$. The functionals $\Phi$ and $\Psi$ satisfy all assumptions requested in Theorem 2.2. Moreover, for $\lambda>0$, the functional $I_{\lambda}$ is coercive. Indeed, fix $0<\varepsilon<\frac{1}{p^{+} \rho^{p^{-}} \lambda \text { meas }(\Omega)}$. From (3.9), there is a function $\rho_{\varepsilon} \in \mathrm{L}^{1}(\Omega)$ such that

$$
F(x, t) \leq \varepsilon t^{p^{-}}+\rho_{\varepsilon}(x),
$$

for every $x \in \Omega$ and $t \in \mathbb{R}$. Taking Proposition 2.4 into account, it follows that, for each $u \in X$ with $\|u\| \geq 1$

$$
\begin{aligned}
\Phi(u)-\lambda \Psi(u) & \geq \frac{1}{p^{+}}\|u\|^{p^{-}}-\lambda \varepsilon \int_{\Omega}|u(x)|^{p^{-}} \mathrm{d} x-\lambda\left\|\rho_{\varepsilon}\right\|_{\mathrm{L}^{1}(\Omega)} \\
& \geq\left(\frac{1}{p^{+}}-\lambda \varepsilon \rho^{p^{-}} \operatorname{meas}(\Omega)\right)\|u\|_{\alpha}^{p^{-}}-\lambda\left\|\rho_{\varepsilon}\right\|_{\mathrm{L}^{1}(\Omega)}
\end{aligned}
$$

It follows that

$$
\lim _{\|u\| \rightarrow+\infty}(\Phi(u)-\lambda \Psi(u))=+\infty
$$


which means the functional $I_{\lambda}=\Phi-\lambda \Psi$ is coercive. Put

$$
\bar{r}=\frac{1}{p^{+}}\left(\frac{\bar{\gamma}}{\rho}\right)^{p^{-}}
$$

and

$$
w_{\bar{\delta}}(t)=\bar{\delta} .
$$

Arguing as in the proof of Theorem 3.1, we obtain that

$$
\rho(\bar{r}) \geq \frac{p^{+}}{\rho^{p^{-}}} \frac{\int_{\Omega} \sup _{|t| \leq \bar{\gamma}} F(x, t) \mathrm{d} x-\int_{\Omega} F(x, \bar{\delta}) \mathrm{d} x}{\bar{\gamma}^{p^{-}}-\rho^{p^{-}} \overline{\boldsymbol{\delta}}^{p^{+}}\|\alpha\|_{\mathrm{L}^{1}(\Omega)}} .
$$

Hence, from our assumption it follows that $\rho(\bar{r})>0$. Therefore, from Theorem 2.2 for each $\lambda>\tilde{\lambda}$, the functional $I_{\lambda}$ admits at least one local minimum $\overline{u_{1}}$ such that

$$
\int_{\Omega} \frac{1}{p(x)}\left(\left|\nabla \overline{u_{1}}(x)\right|^{p(x)}+\sqrt{1+\left|\nabla \overline{u_{1}}(x)\right|^{2 p(x)}}+\alpha(x)\left|\overline{u_{1}}(x)\right|^{p(x)}\right) \mathrm{d} x>\frac{1}{p^{+}}\left(\frac{\bar{\gamma}}{\rho}\right)^{p^{-}} .
$$

The conclusion is achieved.

Remark 3.7. If $f$ is non-negative, then the strong maximum principle ensures that the weak solutions of problem $\left(P_{\lambda}^{f}\right)$ are non-negative (see [30, Lemma 1.1]).

Now, we point out some results in which the function $f$ has separated variables. To be precise, consider the following problem

$$
\left\{\begin{array}{lll}
-\operatorname{div}\left(\left(1+\frac{|\nabla u|^{p(x)}}{\sqrt{1+|\nabla u|^{2 p(x)}}}\right)|\nabla u|^{p(x)-2} \nabla u\right)+\alpha(x)|u|^{p(x)-2} u & \\
=\lambda \theta(x) h(u), & \text { in } \Omega, & \left(P_{\lambda}^{h, \theta}\right) \\
\frac{\partial u}{\partial v}=0, & \text { on } \partial \Omega, &
\end{array}\right.
$$

where $\theta: \Omega \rightarrow \mathbb{R}$ is a non-negative and non-zero function such that $\theta \in \mathrm{L}^{1}(\Omega)$ and $h: \mathbb{R} \rightarrow \mathbb{R}$ is a non-negative continuous function.

Put

$$
H(t)=\int_{0}^{t} h(\xi) \mathrm{d} \xi \quad \text { for all } t \in \mathbb{R} .
$$

The following existence results are consequences of Theorems 3.1-3.6, respectively, by setting $f(x, t)=$ $\theta(x) h(t)$ for every $(x, t) \in \Omega \times \mathbb{R}$.

Theorem 3.8. Assume that $h(0) \neq 0$ and there exist a non-negative constant $\gamma_{1}$ and two positive constants $\gamma_{2}$ and $\delta$, with

$$
\frac{\gamma_{1}}{\rho\|\alpha\|_{1}}<\delta<\sqrt[p^{+}]{\frac{p^{-}}{p^{+}\|\alpha\|_{L^{1}(\Omega)} \rho^{p^{-}}}} \gamma_{2}^{p^{p^{+}}}
$$

such that

$$
\frac{H\left(\gamma_{1}\right)-H(\delta)}{\gamma_{1}^{p^{-}}-\rho^{p^{-}} \delta^{p^{+}}\|\alpha\|_{\mathrm{L}^{1}(\Omega)}}<\frac{H\left(\gamma_{2}\right)-H(\delta)}{\gamma_{2}^{p^{-}}-\rho^{p^{-}} \delta^{p^{+}}\|\alpha\|_{\mathrm{L}^{1}(\Omega)}} .
$$

Furthermore, suppose that there exist constants $v>p^{+}$and $R>0$ such that for all $|\xi| \geq R$ the assumption

$$
0<v H(\xi) \leq \xi h(\xi)
$$


Then, for each $\lambda \in\left(\lambda_{1}, \lambda_{2}\right)$, where

$$
\lambda_{1}=\frac{p^{+}}{\rho^{p^{-}}} \frac{\gamma_{1}^{p^{-}}-\rho^{p^{-}} \delta^{p^{+}}\|\alpha\|_{\mathrm{L}^{1}(\Omega)}}{\|\theta\|_{\mathrm{L}^{1}(\Omega)}\left(H\left(\gamma_{1}\right)-H(\delta)\right)}
$$

and

$$
\lambda_{2}=\frac{p^{+}}{\rho^{p^{-}}} \frac{\gamma_{2}^{p^{-}}-m^{p^{-}} \delta^{p^{+}}\|\alpha\|_{L^{1}(\Omega)}}{\|\theta\|_{L^{1}(\Omega)}\left(H\left(\gamma_{2}\right)-H(\delta)\right)},
$$

problem $\left(P_{\lambda}^{h, \theta}\right)$ admits at least two positive weak solutions $u_{1}$ and $u_{2}$ in $X$ such that

$$
\begin{aligned}
\frac{1}{p^{+}}\left(\frac{\gamma_{1}}{\rho}\right)^{p^{-}} & \leq \int_{\Omega} \frac{1}{p(x)}\left(\left|\nabla u_{1}(x)\right|^{p(x)}+\sqrt{1+\left|\nabla u_{1}(x)\right|^{2 p(x)}}+\alpha(x)\left|u_{1}(x)\right|^{p(x)}\right) \mathrm{d} x \\
& \leq \frac{1}{p^{+}}\left(\frac{\gamma_{2}}{\rho}\right)^{p^{-}} .
\end{aligned}
$$

Theorem 3.9. Assume that $h(0) \neq 0$ and there exist two positive constants $\delta$ and $\gamma$, with

$$
\delta<\sqrt[p^{+}]{\frac{p^{-}}{p^{+}\|\alpha\|_{L^{1}(\Omega)} \rho^{p^{-}}}} \gamma^{p^{-}}
$$

such that

$$
\frac{H(\gamma)}{\gamma^{p^{-}}}<\frac{1}{\rho^{p^{-}}\|\alpha\|_{\mathrm{L}^{1}(\Omega)}} \frac{H(\delta)}{\delta^{p^{+}}}
$$

Furthermore, suppose that the assumption (3.10) holds. Then, for every

$$
\lambda \in\left(\frac{p^{+} \delta^{p^{+}}\|\alpha\|_{\mathrm{L}^{1}(\Omega)}}{\|\theta\|_{\mathrm{L}^{1}(\Omega)} H(\delta)}, \frac{p^{+} \gamma^{p^{-}}}{\rho^{p^{-}}\|\theta\|_{\mathrm{L}^{1}(\Omega)} H(\gamma)}\right),
$$

problem $\left(P_{\lambda}^{h, \theta}\right)$ admits at least two positive weak solutions $u_{1}$ and $u_{2}$ in $X$ such that

$$
0<\int_{\Omega} \frac{1}{p(x)}\left(\left|\nabla u_{1}(x)\right|^{p(x)}+\sqrt{1+\left|\nabla u_{1}(x)\right|^{2 p(x)}}+\alpha(x)\left|u_{1}(x)\right|^{p(x)}\right) \mathrm{d} x \leq \frac{1}{p^{+}}\left(\frac{\gamma}{\rho}\right)^{p^{-}} .
$$

Theorem 3.10. Assume that there exist two positive constants $\bar{\gamma}$ and $\bar{\delta}$ with

$$
\bar{\delta}<\sqrt[p^{+}]{\frac{p^{-}}{p^{+}\|\alpha\|_{L^{1}(\Omega)} \rho^{p^{-}}}} \bar{\gamma}^{p^{-}},
$$

such that

$$
H(\bar{\delta})<H(\bar{\gamma})
$$

and

$$
\lim _{|\xi| \rightarrow+\infty} \frac{h(\xi)}{|\xi|^{p^{-}-1}} \leq 0
$$

Then, for each $\lambda>\bar{\lambda}$, where

$$
\bar{\lambda}:=\frac{p^{+}}{\rho^{p^{-}}} \frac{\rho^{p^{-}} \bar{\delta}^{p^{+}}\|\alpha\|_{L^{1}(\Omega)}-\bar{\gamma}^{p^{-}}}{\|\theta\|_{L^{1}(\Omega)}(H(\bar{\delta})-H(\bar{\gamma}))},
$$

problem $\left(P_{\lambda}^{h, \theta}\right)$ admits at least one positive weak solution $\overline{u_{1}} \in X$ such that

$$
\int_{\Omega} \frac{1}{p(x)}\left(\left|\nabla \overline{u_{1}}(x)\right|^{p(x)}+\sqrt{1+\left|\nabla \overline{u_{1}}(x)\right|^{2 p(x)}}+\alpha(x)\left|\overline{u_{1}}(x)\right|^{p(x)}\right) \mathrm{d} x>\frac{1}{p^{+}}\left(\frac{\bar{\gamma}}{\rho}\right)^{p^{-}} .
$$


Now we illustrate Theorem 3.10 by presenting the following example.

Example 3.11. Let $N=2, \Omega=\left\{\left(x_{1}, x_{2}\right) \in \mathbb{R}^{2} ; x_{1}^{2}+x_{2}^{2}<1\right\} \subset \mathbb{R}^{2}, p\left(x_{1}, x_{2}\right)=e^{1+x_{1}^{2}+x_{2}^{2}}$ for all $\left(x_{1}, x_{2}\right) \in$ $\bar{\Omega}, \alpha\left(x_{1}, x_{2}\right)=\frac{1}{1+x_{1}^{2}+x_{2}^{2}}$ for all $\left(x_{1}, x_{2}\right) \in \bar{\Omega}, \theta\left(x_{1}, x_{2}\right)=x_{1}^{2}+x_{2}^{2}$ for all $\left(x_{1}, x_{2}\right) \in \Omega$ and

$$
h(t)= \begin{cases}2-t^{2}, & |t| \leq 1 \\ 1, & |t|>1\end{cases}
$$

Thus, $p^{-}=e, p^{+}=e^{2}$, meas $(\Omega)=\pi, D=2$,

$$
\begin{gathered}
\|\alpha\|_{L^{1}(\Omega)}=\int_{0}^{2 \pi} \int_{0}^{1} \frac{r}{1+r^{2}} \mathrm{~d} r \mathrm{~d} \theta=\pi \ln (2) \quad \text { and } \quad\|\alpha\|_{\infty}=1, \\
k_{p^{-}}=k_{e} \leq \frac{2}{\sqrt[e]{2}} \max \left\{\frac{1}{\sqrt[e]{\pi \ln 2}}, \frac{2(e-1) \pi}{\pi \ln (2) \sqrt[e]{2 \pi(e-2)}}\right\}=\frac{2}{\sqrt[e]{2 \pi \ln (2)}}
\end{gathered}
$$

and thus, $\rho \leq \frac{2(1+\pi)}{\sqrt[e]{2 \pi \ln 2}}$ and

$$
\|\theta\|_{L^{1}(\Omega)}=\int_{0}^{2 \pi} \int_{0}^{1} r^{3} \mathrm{~d} r \mathrm{~d} \theta=\frac{\pi}{2}
$$

Moreover, $h$ is a non-negative continuous function, $h(0) \neq 0$ and

$$
\lim _{|\xi| \rightarrow+\infty} \frac{h(\xi)}{|\xi|^{p^{-}-1}}=0
$$

By the expression of $h$, we have

$$
H(t)= \begin{cases}t-\frac{2}{3}, & t<-1 \\ 2 t-\frac{t^{3}}{3}, & -1 \leq t \leq 1 \\ t+\frac{2}{3}, & t>1\end{cases}
$$

Thus, by choosing $\bar{\delta}=\frac{2}{e}$ and $\bar{\gamma}=2(1+\pi)$ we clearly observe that (3.12) and (3.13) are satisfied. Indeed,

$$
\bar{\delta}=\frac{2}{e}<\left(\frac{2}{e}\right)^{\frac{1}{e^{2}}} \leq \sqrt[p^{+}]{\frac{p^{-}}{p^{+}\|\alpha\|_{L^{1}(\Omega)} \rho^{p^{-}}}} \bar{\gamma}^{p^{p^{+}}}
$$

and

$$
H(\bar{\delta})=\frac{12 e^{2}-8}{3 e^{3}}<\frac{56}{3}=H(\bar{\gamma}) .
$$

Therefore, by Theorem 3.10 for every $\lambda>\frac{6 e^{5}\left(2^{e^{2}}-(2 e)^{e}(1+\pi)^{e}\right)}{\pi e^{2 e}\left(12 e-2 e^{3}(3 \pi-2)-8\right)}$, the problem

$$
\begin{cases}-\operatorname{div}\left(\left(1+\frac{\left|\nabla u\left(x_{1}, x_{2}\right)\right|^{1+|x|}}{\sqrt{1+\left|\nabla u\left(x_{1}, x_{2}\right)\right|^{2 e^{1+|x|}}}}\right)\left|\nabla u\left(x_{1}, x_{2}\right)\right|^{e^{1+|x|^{2}}-2} \nabla u\left(x_{1}, x_{2}\right)\right) & \\ =\lambda|x|^{2} h\left(u\left(x_{1}, x_{2}\right)\right), & \text { in } \Omega \\ \frac{\partial u}{\partial v}=0 & \text { on } \partial \Omega\end{cases}
$$

has at least one positive weak solution

$$
\overline{u_{1}} \in\left\{u \in \mathrm{L}^{e^{1+|x|^{2}}}(\Omega):|\nabla u| \in \mathrm{L}^{e^{1+|x|^{2}}}(\Omega)\right\}
$$


such that

$$
\begin{aligned}
& \iint_{|x|^{2}<1} \frac{1}{e^{1+|x|^{2}}}\left(\left|\nabla u_{1}\left(x_{1}, x_{2}\right)\right|^{e^{1+|x|^{2}}}+\sqrt{1+\left|\nabla u_{1}\left(x_{1}, x_{2}\right)\right|^{2 e^{1+|x|^{2}}}}\right. \\
& \left.\quad+\frac{\left|u_{1}\left(x_{1}, x_{2}\right)\right|^{1+|x|^{2}}}{1+|x|^{2}}\right) \mathrm{d} x_{1} \mathrm{~d} x_{2}>\frac{2 \pi \ln (2)}{e^{2}}-1
\end{aligned}
$$

where $|x|=\sqrt{x_{1}^{2}+x_{2}^{2}}$.

A further consequence of Theorem 3.1 is the following existence result.

Theorem 3.12. Assume that $h(0) \neq 0$ and

$$
\lim _{\xi \rightarrow 0^{+}} \frac{h(\xi)}{\xi p^{-}-1}=+\infty
$$

Furthermore, suppose that the assumption (3.10) holds. Then, for every $\lambda \in\left(0, \lambda_{\gamma}^{\star}\right)$, where

$$
\lambda_{\gamma}^{\star}:=\frac{p^{+}}{\rho^{p^{-}}\|\theta\|_{L^{1}(\Omega)}} \sup _{\gamma>0} \frac{\gamma^{p^{-}}}{H(\gamma)},
$$

problem $\left(P_{\lambda}^{h, \theta}\right)$ admits at least two positive weak solutions in $X$.

Proof. Fix $\lambda \in] 0, \lambda_{\gamma}^{\star}[$. Then there is $\gamma>0$ such that

$$
\lambda<\frac{p^{+}}{\rho^{p^{-}}\|\theta\|_{L^{1}(\Omega)}} \sup _{\gamma>0} \frac{\gamma^{p^{-}}}{H(\gamma)} .
$$

From (3.14) there exists a positive constant $\delta$ with

$$
\delta<\sqrt[p^{+}]{\frac{p^{-}}{p^{+}\|\alpha\|_{L^{1}(\Omega)} \rho^{p^{-}}}} \gamma^{\frac{p^{-}}{p^{+}}}
$$

such that

$$
\frac{1}{\lambda}<\frac{\|\theta\|_{\mathrm{L}^{1}(\Omega)} H(\delta)}{\rho^{p^{-}} \delta^{p^{+}}\|\alpha\|_{\mathrm{L}^{1}(\Omega)}} .
$$

Therefore, we can use Theorem 3.4 to complete the proof.

Remark 3.13. Theorem 1.1 immediately follows from Theorem 3.12.

Now we illustrate Theorem 3.12 by presenting the following example.

Example 3.14. Let $N=3, \Omega=\left\{\left(x_{1}, x_{2}, x_{3}\right) \in \mathbb{R}^{2} ; x_{1}^{2}+x_{2}^{2}+x_{3}^{2}<1\right\} \subset \mathbb{R}^{3}, p\left(x_{1}, x_{2}, x_{3}\right)=4+x_{1}^{2}+x_{2}^{2}+x_{3}^{2}$ for all $\left(x_{1}, x_{2}, x_{3}\right) \in \bar{\Omega}, \alpha\left(x_{1}, x_{2}, x_{3}\right)=x_{1}^{2}+x_{2}^{2}+x_{3}^{2}$ for all $\left(x_{1}, x_{2}, x_{3}\right) \in \bar{\Omega}, \theta\left(x_{1}, x_{2}\right)=1$ for all $\left(x_{1}, x_{2}, x_{3}\right) \in$ $\Omega$ and

$$
h(t)= \begin{cases}20+t^{20}, & |t| \geq 1 \\ 22-t^{2}, & |t|<1 .\end{cases}
$$

Direct calculations give $p^{-}=4, p^{+}=5$, meas $(\Omega)=\frac{4 \pi}{3}, D=2$,

$$
\begin{gathered}
\|\alpha\|_{\mathrm{L}^{1}(\Omega)}=\int_{0}^{2 \pi} \int_{0}^{\pi} \int_{0}^{1} r^{4} \sin \phi \mathrm{d} r \mathrm{~d} \phi \mathrm{d} \theta=\frac{4 \pi}{5} \text { and }\|\alpha\|_{\infty}=1 \\
k_{p^{-}}=k_{4} \leq \frac{2}{\sqrt[4]{2}} \max \left\{\sqrt[4]{\frac{5}{4 \pi}}, \frac{5}{\sqrt[4]{4 \pi}}\right\}=\frac{10}{\sqrt[4]{8 \pi}}
\end{gathered}
$$


and thus, $\rho \leq \frac{10}{\sqrt[4]{8 \pi}}\left(1+\frac{4 \pi}{3}\right)$. Also $\|\theta\|_{\mathrm{L}^{1}(\Omega)}=\frac{4 \pi}{3}$,

$$
\lim _{\gamma \rightarrow+\infty} \frac{\gamma^{p^{-}}}{H(\gamma)}=\lim _{\gamma \rightarrow \infty} \frac{\gamma^{4}}{22 \gamma-\frac{\gamma^{3}}{3}}=+\infty
$$

and

$$
\lim _{\xi \rightarrow 0^{+}} \frac{h(\xi)}{\xi}=+\infty
$$

Moreover, since

$$
\lim _{|\xi| \rightarrow+\infty} \frac{\xi h(\xi)}{H(\xi)}=\lim _{|\xi| \rightarrow+\infty} \frac{20 t+t^{21}}{20 t+\frac{t^{21}}{21}}=21>5=p^{+}
$$

by choosing $v=21>5=p^{+}$we can choose $R>1$ such that the assumption (3.10) is fulfilled. Hence, by applying Theorem 3.12, for every $\lambda>0$ the problem

$$
\begin{cases}-\operatorname{div}\left(\left(1+\frac{\left|\nabla u\left(x_{1}, x_{2}, x_{3}\right)\right|^{4+|x|^{2}}}{\sqrt{1+\left|\nabla u\left(x_{1}, x_{2}, x_{3}\right)\right|^{8+2|x|^{2}}}}\right)|\nabla u|^{|x|^{2}+2} \nabla u\left(x_{1}, x_{2}, x_{3}\right)\right) & \\ =\lambda h\left(u\left(x_{1}, x_{2}, x_{3}\right)\right), & \text { in } \Omega \\ \frac{\partial u}{\partial v}=0 & \text { on } \partial \Omega\end{cases}
$$

where $|x|=\sqrt{x_{1}^{2}+x_{2}^{2}+x_{3}^{2}}$, has at least two positive weak solutions.

Remark 3.15. The non-triviality of the second weak solution ensured by Theorem 3.12 can be achieved also in the case $h(0)=0$ requiring the extra condition at zero, that is, there are a non-empty open set $D \subseteq \Omega$ and a set $B \subset D$ of positive Lebesgue measure such that

$$
\limsup _{\xi \rightarrow 0^{+}} \frac{\operatorname{essinf}_{t \in B} \theta(t) \cdot H(\xi)}{|\xi|^{-}}=+\infty
$$

and

$$
\liminf _{\xi \rightarrow 0^{+}} \frac{\operatorname{essinf}_{t \in D} \theta(t) \cdot H(\xi)}{|\xi|^{p^{-}}}>-\infty .
$$

Indeed, arguing as in $[5,31]$ let $0<\bar{\lambda}<\lambda^{*}$, where

$$
\lambda^{*}=\frac{p^{+}}{\rho^{p^{-}}\|\theta\|_{L^{1}(\Omega)}} \sup _{\gamma>0} \frac{\gamma^{p^{-}}}{H(\gamma)} \text {. }
$$

Then, there exists $\bar{\gamma}>0$ such that

$$
\bar{\lambda} \frac{\rho^{p^{-}}\|\theta\|_{\mathrm{L}^{1}(\Omega)}}{p^{+}}<\frac{\bar{\gamma}^{p^{-}}}{H(\bar{\gamma})} .
$$

Let $\Phi$ and $\Psi$ be as given in (2.4) and (2.5), respectively. Due to Theorem 3.12, for every $\lambda \in(0, \bar{\lambda})$ there exists a critical point of $I_{\lambda}=\Phi-\lambda \Psi$ such that $u_{\lambda} \in \Phi^{-1}\left(-\infty, r_{\lambda}\right)$, where $r_{\lambda}=\frac{1}{p^{+}}\left(\frac{\bar{\gamma}}{\rho}\right)^{p^{-}}$. In particular, $u_{\lambda}$ is a global minimum of the restriction of $I_{\lambda}$ to $\Phi^{-1}\left(-\infty, r_{\lambda}\right)$. We will prove that the function $u_{\lambda}$ cannot be trivial. Let us show that

$$
\limsup _{\|u\| \rightarrow 0^{+}} \frac{\Psi(u)}{\Phi(u)}=+\infty \text {. }
$$

Owing to the assumptions (3.15) and (3.16), we can consider a sequence $\left\{\xi_{n}\right\} \subset \mathbb{R}^{+}$converging to zero and two constants $\sigma, \kappa$ (with $0<\sigma<1$ ) such that

$$
\lim _{n \rightarrow+\infty} \frac{e s s \operatorname{sinf}_{t \in B} \theta(t) \cdot H\left(\xi_{n}\right)}{\left|\xi_{n}\right|^{p^{-}}}=+\infty
$$


and

$$
\text { ess } \inf _{t \in D} \theta(t) \cdot H(\xi) \geq \kappa|\xi|^{p^{-}}
$$

for every $\xi \in[0, \sigma]$. We consider a set $\mathscr{G} \subset B$ of positive measure and a function $v \in X$ such that

$\left(k_{1}\right) v(t) \in[0,1]$ for every $t \in \Omega$,

$\left(k_{2}\right) v(t)=1$ for every $t \in \mathscr{G}$,

$\left(k_{3}\right) v(t)=0$ for every $x \in \Omega \backslash D$.

Hence, fix $N>0$ and consider a real positive number $\eta$ with

$$
N<\frac{p^{+} \eta \operatorname{meas}(\mathscr{G})+p^{+} \kappa \int_{D \backslash \mathscr{G}}|v(t)|^{p^{-}} \mathrm{d} t}{\|v\|_{\alpha}^{p^{-}}} .
$$

Then, there is $n_{0} \in \mathbb{N}$ such that $\xi_{n}<\sigma$ and

$$
\operatorname{ess} \inf _{t \in B} \theta(t) \cdot H\left(\xi_{n}\right) \geq \eta\left|\xi_{n}\right|^{p^{-}}
$$

for every $n>n_{0}$. Now, for every $n>n_{0}$, by considering the properties of the function $v$ (that is $0 \leq$ $\xi_{n} v(t)<\sigma$ for $n$ large enough), one has

$$
\begin{aligned}
\frac{\Psi\left(\xi_{n} v\right)}{\Phi\left(\xi_{n} v\right)} & \geq \frac{\int_{\mathscr{G}} \theta(t) \cdot H\left(\xi_{n}\right) \mathrm{d} t+\int_{D \backslash \mathscr{G}} \theta(t) \cdot H\left(\xi_{n} v(t)\right) \mathrm{d} t}{\Phi\left(\xi_{n} v\right)} \\
& >\frac{p^{+} \eta \operatorname{meas}(\mathscr{G})+p^{+} \kappa \int_{D \backslash \mathscr{G}}|v(t)|^{p^{-}} \mathrm{d} t}{\|v\|_{\alpha}^{p^{-}}}>N .
\end{aligned}
$$

Since $N$ could be arbitrarily large, we get

$$
\lim _{n \rightarrow \infty} \frac{\Psi\left(\xi_{n} v\right)}{\Phi\left(\xi_{n} v\right)}=+\infty
$$

from which (3.17) clearly follows. So, there exists a sequence $\left\{\omega_{n}\right\} \subset X$ strongly converging to zero such that, for $n$ large enough, $\omega_{n} \in \Phi^{-1}(-\infty, r)$ and

$$
I_{\lambda}\left(\omega_{n}\right)=\Phi\left(\omega_{n}\right)-\lambda \Psi\left(\omega_{n}\right)<0 .
$$

Since $u_{\lambda}$ is a global minimum of the restriction of $I_{\lambda}$ to $\Phi^{-1}(-\infty, r)$, we obtain

$$
I_{\lambda}\left(u_{\lambda}\right)<0
$$

so that $u_{\lambda}$ is not trivial.

Next, as a consequence of Theorems 3.9 and 3.10, the following theorem of the existence of three solutions is obtained.

Theorem 3.16. Suppose that $h(0) \neq 0$ and

$$
\limsup _{|\xi| \rightarrow+\infty} \frac{H(\xi)}{|\xi| p^{-}} \leq 0
$$

Moreover, assume that there exist four positive constants $\gamma, \delta, \bar{\gamma}$ and $\bar{\delta}$ with

$$
\delta<\sqrt[p^{+}]{\frac{p^{-}}{p^{+}\|\alpha\|_{\mathrm{L}^{1}(\Omega)} \rho^{p^{-}}}} \gamma^{\frac{p^{-}}{p^{+}}}<\sqrt[p^{+}]{\frac{p^{-}}{p^{+}\|\alpha\|_{\mathrm{L}^{1}(\Omega)} \rho^{p^{-}}}} \bar{\gamma}^{{\frac{p}{p^{+}}}^{p^{+}}}<\bar{\delta}
$$


such that (3.11) and (3.13) hold, and

$$
\frac{H(\gamma)}{\gamma^{p^{-}}}<\frac{H(\bar{\delta})-H(\bar{\gamma})}{\bar{\gamma}^{p^{-}}-\rho^{p^{-}} \bar{\delta}^{p^{+}}\|\alpha\|_{\mathrm{L}^{1}(\Omega)}}
$$

are satisfied. Then, for each

$$
\lambda \in \Lambda=\left(\max \left\{\tilde{\lambda}, \frac{\rho^{p^{-}} \delta^{p^{+}}\|\alpha\|_{\mathrm{L}^{1}(\Omega)}}{\|\theta\|_{\mathrm{L}^{1}(\Omega)} H(\delta)}\right\}, \frac{p^{+}}{\rho^{p^{-}}} \frac{\gamma^{p^{-}}}{\|\theta\|_{\mathrm{L}^{1}(\Omega)} H(\gamma)}\right),
$$

problem $\left(P_{\lambda}^{h, \theta}\right)$ admits at least three positive weak solutions $u_{1}, \bar{u}_{1}$ and $u_{3}$ such that

$$
\int_{\Omega} \frac{1}{p(x)}\left(\left|\nabla u_{1}(x)\right|^{p(x)}+\sqrt{1+\left|\nabla u_{1}(x)\right|^{2 p(x)}}+\alpha(x)\left|u_{1}(x)\right|^{p(x)}\right) \mathrm{d} x \leq \frac{1}{p^{+}}\left(\frac{\gamma}{\rho}\right)^{p^{-}}
$$

and

$$
\int_{\Omega} \frac{1}{p(x)}\left(\left|\nabla \overline{u_{1}}(x)\right|^{p(x)}+\sqrt{1+\left|\nabla \overline{u_{1}}(x)\right|^{2 p(x)}}+\alpha(x)\left|\overline{u_{1}}(x)\right|^{p(x)}\right) \mathrm{d} x>\frac{1}{p^{+}}\left(\frac{\bar{\gamma}}{\rho}\right)^{p^{-}} .
$$

Proof. First, in view of (3.20), we have $\Lambda \neq \emptyset$. Next, fix $\lambda \in \Lambda$. Employing Theorem 3.9, there is a positive weak solution $u_{1}$ such that

$$
\int_{\Omega} \frac{1}{p(x)}\left(\left|\nabla u_{1}(x)\right|^{p(x)}+\sqrt{1+\left|\nabla u_{1}(x)\right|^{2 p(x)}}+\alpha(x)\left|u_{1}(x)\right|^{p(x)}\right) \mathrm{d} x \leq \frac{1}{p^{+}}\left(\frac{\gamma}{\rho}\right)^{p^{-}},
$$

which is a local minimum for the associated functional $I_{\lambda}$, while Theorem 3.10 ensures a positive weak solution $\overline{u_{1}}$ such that

$$
\int_{\Omega} \frac{1}{p(x)}\left(\left|\nabla \overline{u_{1}}(x)\right|^{p(x)}+\sqrt{1+\left|\nabla \overline{u_{1}}(x)\right|^{2 p(x)}}+\alpha(x)\left|\overline{u_{1}}(x)\right|^{p(x)}\right) \mathrm{d} x>\frac{1}{p^{+}}\left(\frac{\bar{\gamma}}{\rho}\right)^{p^{-}},
$$

which is a local minimum for $I_{\lambda}$. Arguing as in the proof of Theorem 3.6, from the condition (3.19) we observe that the functional $I_{\lambda}$ is coercive. Then it satisfies the (P.S) condition. Hence, the conclusion follows from the mountain pass theorem as given by Pucci and Serrin (see [23]).

The following existence result is a consequence of Theorem 3.16.

Theorem 3.17. Assume that $h(0) \neq 0$,

$$
\limsup _{\xi \rightarrow 0^{+}} \frac{H(\xi)}{\xi p^{-}}=+\infty
$$

and

$$
\limsup _{\xi \rightarrow+\infty} \frac{H(\xi)}{\xi p^{-}}=0
$$

Furthermore, suppose that there exist two positive constants $\bar{\gamma}$ and $\bar{\delta}$ with

$$
\sqrt[p^{+}]{\frac{p^{-}}{p^{+}\|\alpha\|_{\mathrm{L}^{1}(\Omega)} \rho^{p^{-}}}} \bar{\gamma}^{p^{p^{-}}}<\bar{\delta}
$$

such that

$$
\frac{H(\bar{\gamma})}{\bar{\gamma}^{p^{-}}}<\frac{p^{+}}{\rho^{2 p^{-}}\|\alpha\|_{\mathrm{L}^{1}(\Omega)}^{2}} \frac{H(\bar{\delta})}{\overline{\boldsymbol{\delta}}^{p^{+}}}
$$


Then, for each

$$
\lambda \in\left(\frac{\rho^{p^{-}}\|\alpha\|_{\mathrm{L}^{1}(\Omega)}}{\|\theta\|_{\mathrm{L}^{1}(\Omega)}} \frac{\bar{\delta}^{p^{+}}}{H(\bar{\delta})}, \frac{p^{+}}{\rho^{p^{-}}\|\alpha\|_{\mathrm{L}^{1}(\Omega)}} \frac{\bar{\gamma}^{p^{-}}}{\|\theta\|_{\mathrm{L}^{1}(\Omega)} H(\bar{\gamma})}\right),
$$

problem $\left(P_{\lambda}^{h, \theta}\right)$ admits at least three positive weak solutions.

Proof. We easily observe from (3.22) that condition (3.19) is satisfied. Moreover, by choosing $\delta$ small enough and $\gamma=\bar{\gamma}$, one can drive condition (3.11) from (3.21) as well as the conditions (3.13) and (3.20) from (3.24). Hence, the conclusion follows from Theorem 3.16.

Remark 3.18. Theorem 1.2 immediately follows from Theorem 3.17.

Finally, we present one application of Theorem 3.17 as follows.

Example 3.19. Let $N>1, p^{-}>N$ and $\Omega \subset \mathbb{R}^{N}$ be an open bounded domain with smooth boundary $\partial \Omega$ such that

$$
\operatorname{meas}(\Omega) \leq \frac{p^{-}-N}{p^{-}-1}\left(\frac{N}{\operatorname{diam}(\Omega)^{p^{-}}}\right)^{\frac{1}{p^{-}-1}}
$$

Also let

$$
\begin{aligned}
& p^{+}>\frac{2^{2 p^{-}-1}(1+\text { meas }(\Omega))^{2 p^{-}}}{\left(2-\frac{\pi}{4}\right)(e-1)^{p^{-}-1}}, \\
& h(t)=1+\frac{t^{2}}{1+t^{2}}, \quad \text { for all } t \in \mathbb{R}
\end{aligned}
$$

and $\|\theta\|_{\mathrm{L}^{1}(\Omega)}=1$. Now, by choosing $\alpha$ in such a way that $\|\alpha\|_{\mathrm{L}^{1}(\Omega)}=\|\alpha\|_{\infty}=1$ we have $\rho<2^{\frac{p^{-}-1}{p^{-}}}(1+\operatorname{meas}(\Omega))$, $h$ is a non-negative continuous function, $h(0) \neq 0$,

$$
\begin{gathered}
H(t)=2 t-\arctan t \quad \text { for all } t \in \mathbb{R}, \\
\lim _{\xi \rightarrow 0^{+}} \frac{H(\xi)}{\xi^{p^{-}}}=\lim _{\xi \rightarrow 0^{+}} \frac{2 \xi-\arctan \xi}{\xi^{-}}=+\infty
\end{gathered}
$$

and

$$
\lim _{\xi \rightarrow+\infty} \frac{H(\xi)}{\xi p^{-}}=\lim _{\xi \rightarrow+\infty} \frac{2 \xi-\arctan \xi}{\xi^{-}}=0 .
$$

Moreover, by choosing $\bar{\gamma}=e$ and $\bar{\delta}=1$, we see that (3.23) and (3.24) are satisfied. Hence, by applying Theorem 3.17, for every

$$
\lambda \in\left(\frac{2^{p^{-}-1}(1+\operatorname{meas}(\Omega))^{p^{-}}}{\operatorname{meas}(\Omega)\left(2-\frac{\pi}{4}\right)}, \frac{p^{+}(e-1)^{p^{-}-1}}{2^{p^{-}} \operatorname{meas}(\Omega)(1+\operatorname{meas}(\Omega))^{p^{-}}}\right),
$$

the problem

$$
\begin{cases}-\operatorname{div}\left(\left(1+\frac{|\nabla u|^{p(x)}}{\sqrt{1+|\nabla u|^{2 p(x)}}}\right)|\nabla u|^{p(x)-2} \nabla u\right)+\alpha(x)|u|^{p(x)-2} u & \\ =\lambda \theta(x)\left(1+\frac{u^{2}(x)}{1+u^{2}(x)}\right), & \text { in } \Omega, \\ \frac{\partial u}{\partial v}=0, & \text { on } \partial \Omega\end{cases}
$$

has at least three positive weak solutions. 
Remark 3.20. We point out that the same statements of the above given results can be obtained by considering

$$
M(t)=b_{1}+b_{2} t, \quad \text { for } t \in[l, \kappa],
$$

where $b_{1}, b_{2}, \imath$ and $\kappa$ are positive numbers. Arguing as in the proof of Theorem 3.1, the first and second solutions can be obtained.

Remark 3.21. We note that the same statements of the above given results can be obtained by considering $p(x) \equiv p$. In this case, the $p(x)$-Laplacian operator is the well-known $p$-Laplacian. The $p(x)$ Laplacian is more complicated nonlinearities than $p$-Laplacian. For example, the $p$-Laplacian operator is $(p-1)$ homogeneous, that is, $\Delta_{p}(s u)=s^{p-1} \Delta_{p} u$ for every $s>0$, but the $p(x)$-Laplacian operator is not homogeneous.

\section{REFERENCES}

[1] X.L. Fan, S.G. Deng, Multiplicity of positive solutions for a class of inhomogeneous Neumann problems involving the $p(x)$-Laplacian, NoDEA Nonlinear Differential Equations Appl. 16 (2009), 255-271.

[2] S.G. Deng, A local mountain pass theorem and applications to a double perturbed $p(x)$-Laplacian equations, Appl. Math. Comput. 211 (2009), 234-241.

[3] F. Cammaroto, L. Vilasi, Multiplicity results for a Neumann boundary value problem involving the $p(x)$-laplacian, Taiwanese J. Math. 16 (2012), 621-634.

[4] S. Heidarkhani, B. Ge, Critical points approaches to elliptic problems driven by a $p(x)$-Laplacian, Ukrainian Math. J. 66 (2015), 1883-1903.

[5] G. A. Afrouzi, A. Hadjian, G. Molica Bisci, Remarks for one-dimensional mean curvature problems through a local minimization principle, Adv. Nonlinear Anal. 2 (2013), 427-441.

[6] C. Bereanu, J. Mawhin, Boundary value problems with non-surjective $\phi$-Laplacian and one-sided bounded nonlinearity, Adv. Differential Equations, 11 (2006), 35-60.

[7] F. Faraci, A note on the existence of infinitely many solutions for the one dimensional prescribed curvature equation, Stud. Univ. Babeş-Bolyai Math. 55 (2010), 83-90.

[8] H. Pan, One-dimensional prescribed mean curvature equation with exponential nonlinearity, Nonlinear Anal. 70 (2009), 999-1010.

[9] C.V. Coffman, W.K. Ziemer, A prescribed mean curvature problem on domains without radial symmetry, SIAM J. Math. Anal. 22 (1991), 982-990.

[10] M. Nakao, A bifurcation problem for a quasi-linear elliptic boundary value problem, Nonlinear Anal. 14 (1990), $251-262$.

[11] P. Habets, P. Omari, Positive solutions of an indefinite prescribed mean curvature problem on a general domain, Adv. Nonlinear Stud. 4 (2004), 1-13.

[12] V.K. Le, Some existence results on nontrivial solutions of the prescribed mean curvature equation, Adv. Nonlinear Stud. 5 (2005), 133-161

[13] M. Marzocchi, Multiple solutions of quasilinear equations involving an area-type term, J. Math. Anal. Appl. 196 (1995), 1093-1104.

[14] F. Obersnel, P. Omari, Positive solutions of the Dirichlet problem for the prescribed mean curvature equation, J. Differential Equations 249 (2010), 1674-1725.

[15] S. Shokooh, Existence and multiplicity results for elliptic equations involving the p-Laplacian-like, Ann. Univ. Craiova, Mathematics and Computer Science Series 44 (2017), 249-258.

[16] M.M. Rodrigues, Multiplicity of solutions on a nonlinear eigenvalue problem for $p(x)$-Laplacian-like operators, Mediterr. J. Math. 9 (2012), 211-223.

[17] K.C. Chang, Critical Point Theory and Applications, Shanghai Scientific and Technology Press, Shanghai, 1986.

[18] M. Willem, Minimax Theorems, Birkhauser, Basel, 1996.

[19] G. Bin, On superlinear $p(x)$-Laplacian-like problem without Ambrosetti and Rabinowitz condition, Bull. Korean Math. Soc. 51 (2014), 409-421. 
[20] Q.M. Zhou, On the superlinear problems involving $p(x)$-Laplacian-like operators without AR-condition, Nonlinear Anal. 21 (2015), 161-169.

[21] G. Bonanno, A critical point theorem via the Ekeland variational principle, Nonlinear Anal. 75 (2012), 2992-3007.

[22] A. Ambrosetti, P.H. Rabinowitz, Dual variational methods in critical point theory and applications, J. Funct. Anal. 14 (1973), 349-381.

[23] P. Pucci, J. Serrin, A mountain pass theorem, J. Differential Equations 60 (1985), 142-149.

[24] B. Ricceri, A general variational principle and some of its applications, J. Comput. Appl. Math. 113 (2000), $401-410$.

[25] G. D’Aguì, Multiplicity results for nonlinear mixed boundary value problem, Bound. Value Probl. 2012 (2012), 1-12.

[26] X. Fan, D. Zhao, On the spaces $L^{p(x)}(\Omega)$ and $W^{m, p(x)}(\Omega)$, J. Math. Anal. Appl. 263 (2001), 424-446.

[27] O. Kováčik, J. Rákosník, On the spaces $L^{p(x)}(\Omega)$ and $W^{1, p(x)}(\Omega)$, Czechoslovak Math. J. 41 (1991), 592-618.

[28] G. Bonanno, P. Candito, Three solutions to a Neumann problem for elliptic equations involving the $p$-Laplacian, Arch. Math. (Basel) 80 (2003), 424-429.

[29] P.H. Rabinowitz, Minimax methods in critical point theory with applications to differential equations, CBMS Reg. Conf. Ser. Math., Vol. 65, Amer. Math. Soc. Providence, RI, 1986.

[30] X.L. Fan, Q.H. Zhang, Y.Z. Zhao, A strong maximum principle for $p(x)$-Laplace equations, Chin. J. Contemp. Math. 24 (2003), 277-282.

[31] G. Bonanno, Relations between the mountain pass theorem and local minima, Adv. Nonlinear Anal. 1 (2012), 205-220. 